\title{
The Wolf and the Caribou: Coexistence of Decentralized Economies and Competitive Markets
}

\author{
Andreas Freund ${ }^{1}$ and Danielle Stanko ${ }^{2, *}$ \\ 1 Head of Technology, TCS Blockchain, Tata Consultancy Services LLC, 101 Park Avenue, \\ New York, NY 10178, USA; andreas.freund@tcs.com \\ 2 TCS Blockchain, Tata Consultancy Services LLC, 101 Park Avenue, New York, NY 10178, USA \\ * Correspondence: Danielle.s@tcs.com
}

Received: 31 March 2018; Accepted: 16 May 2018; Published: 23 May 2018

\begin{abstract}
Starting with BitTorrent and then Bitcoin, decentralized technologies have been on the rise over the last $15+$ years, gaining significant momentum in the last $2+$ years with the advent of platform ecosystems such as the Blockchain platform Ethereum. New projects have evolved from decentralized games to marketplaces to open funding models to decentralized autonomous organizations. The hype around cryptocurrency and the valuation of innovative projects drove the market cap of cryptocurrencies to over a trillion dollars at one point in 2017. These high valued technologies are now enabling something new: globally scaled and decentralized business models. Despite their valuation and the hype, these new business ecosystems are frail. This is not only because the underlying technology is rapidly evolving, but also because competitive markets see a profit opportunity in exponential cryptocurrency returns. This extracts value from these ecosystems, which could lead to their collapse, if unchecked. In this paper, we explore novel ways for decentralized economies to protect themselves from, and coexist with, competitive markets at a global scale utilizing decentralized technologies such as Blockchain.
\end{abstract}

Keywords: Blockchain; cryptocurrency; decentralization; ecosystems; game theory

\section{Introduction}

We live in a fascinating time in human history. Humanity is rapidly approaching a "Singularity" as Ray Kurzweil put it in his book "The Singularity is Near" (Kurzweil 2005), referencing the point in human history when artificial intelligence agents will be more intelligent than the entire human race. This "Singularity" is driven by the exponential nature of Moore's Law in the underlying technologies such as Big Data, Cloud, IoT, and Social Media. As these technologies rapidly connect more and more people globally, humanity is also evolving as new needs drive new technologies to fulfill those needs (Beck et al. 2018). Today's hyperconnectedness exposes both belief and need dichotomies, "Us" vs. "Other", of billions of people at a global scale in real time, and, therefore, also our subconscious reactions to the "Other" that have been shaped by millions of years of evolutionary development. This global real time phenomenon is leading to greater, deeper, and more extensive conflicts globally from the Global Financial Crisis to the Arab Spring to Global Warming to the rise of global extremist terrorism and local armed conflicts. This in turn leads to rising global anxiety, disenfranchisement, and anger with an intensifying deep mistrust in traditional, centralized trust structures such as government at all levels, global companies, and even NGOs. As a consequence, humanity has started to search for both technological and organizational solutions also at a global scale and in real time. This has given rise over the last nearly 20 years to an entirely new class of technologies and organizational structures such as Bittorrent, Bitcoin, Ethereum, IPFS, and IOTA etc., with a combined valuation of roughly \$1Tn today, and peer to peer (p2p) business models such as Sensorica, Teambrella, Backfeed, the DAO, Augur 
and purpose driven social networks, most importantly the decentralized organizational structure of the Arab Spring in 2011. Because centralized entities commonly fail to address current global problems, this class of technologies and organizations abandons the primacy of centralization that focuses solely on the self, or "I". This psychological "I" focus has been the predominant paradigm of both society and technology since the dawn of time due to our physiological limitation to maintain very large numbers of trust relationships (Dunbar 1992). These technologies and organizations put decentralization at the center, where the psychological focus on "We" manifests as a global tribe that trusts one another, is mutually supportive, collaborative, and sustainable (Beck et al. 2018). This is a fundamental psychological paradigm shift that has never happened before in human history (Kish n.d.). This is yet another, and in fact, fundamental belief dichotomy that seems to be irreconcilable, promising more and even deeper conflict between "I" and "We" organizations.

While not claiming to have resolved the basic dichotomy, in this paper, the authors propose novel ways how decentralized, collaborative organizational structures can coexist and thrive with the current predominant centralized organizational structures without being destroyed. We will first briefly document the rise of decentralized socioeconomic models to help the reader understand the predecessors of these relatively new types of models, then detail the main differences between decentralized socioeconomic and competitive markets to highlight expected behavior when the two types of markets interact. The market comparison section also includes a recommended construct for decentralized socioeconomic models, characterized as a three zone model embodying specific protective design considerations. We detail the types of value extraction attacks to be expected from the coexistence of decentralized socioeconomic and competitive organizations, and mention the role of market interfaces in the proliferation of the former. Then, using case studies, we illustrate ways that these two models currently interact, and finally make several proposals that can be employed to protect decentralized economies from extractive market forces ${ }^{1}$ while enabling a mutually beneficial coexistence.

\section{Methodology}

The methodology used to construct the three zoned model was based on empirical evidence of case studies and additional commons based organizations in operation, studies related to behavioral economics, and logical arguments based on game theory. The four proposals were developed using empirical evidence and articles related to Blockchain technology and decentralized governance, open value accounting and theories of value, as well as monetary and fiscal policy. Attack mitigation considerations were largely based on empirical evidence of cryptocurrencies, cryptography, security proofs of consensus algorithms, and logical arguments based on game theory.

\section{The Rise of Decentralized Socioeconomic Models}

The "We" mindset of viewing the world as a global tribe has been academically understood for decades (Graves 2005), but was only observed on occasion. This mindset is growing, as evidenced by over 600 examples $^{2}$ of decentralized technology organizations related to global well-being that appeared within the last two years (Startup Tracker 2018). There is a growing sentiment that business should be optimized for both profit and purpose, bringing socioeconomics closer to business considerations than it had been during the industrial revolution and under capitalistic market ideology. When socioeconomics are introduced into capitalist-era business, two predominant changes happen. One is that power and rewards of business move away from a small group of people and are distributed

\footnotetext{
Meaning non-members profit, but at a net loss to the decentralized market.

Blockchain projects related to supply chain \& logistics, provenance \& notary, payments, legal, audit \& tax, Internet of Things, infrastructure, identity \& reputation, compliance \& security, data analytics, financial services, and governance \& transparency were considered to be "global well-being purposes," which comprised 50\% of the 1352 Blockchain startups listed (Startup Tracker 2018).
} 
more evenly among all contributors in the cooperative model (decentralization of power and rewards), bringing about more value equality. The other change is business's purpose transitioning from filling a profitable market void regardless of the impact, to filling unmet human needs in a profitable way with positive social impact, generally referred to as the "social enterprise" (SE) (Dees and Anderson 2007). With the exception of some early cooperatives providing access to electricity or business resources for farmers (National Association of Housing Cooperatives n.d.), these two characteristics have historically existed independently.

Decentralized business models first emerged in the 1800s with agricultural cooperatives that pooled small farms' resources to achieve economies of scale for marketing, supplies, and services (Ortmann and King 2007). The cooperative model extended to other businesses near the early 1900s by providing electricity, financial services through credit unions, and even housing to members (National Association of Housing Cooperatives n.d.). Another hundred years later in 2005, the United States National Cooperative Business Association estimated that there were 750,000 cooperatives globally (Ortmann and King 2007). Nine years later, after the Global Financial Crisis brought a wave of distrust in centralized institutions, this number had more than tripled to 2.6 million in 2015 (Dave Grace \& Associates 2014). Parallel to the rise of decentralized business models, SEs were also working their way into the business landscape.

Social enterprises grew in the United States during the Reagan administration when government funding was reduced for non-profits, forcing non-profits and people to find new mechanisms to meet social needs. The successful mechanism for replacing lost government funding became earning revenue like a business. In 1982, the first international conference on the topic was held and $250 \mathrm{C}-$ level executives attended (Boschee 2010). In 2006, the B Lab was founded to support and help the SE concept proliferate. By 2015, 1358 corporations were registered as "For-Benefit Corporations" (Harriman 2015).

Technology that connects people instantaneously and across geographies is now becoming a new tool to proliferate decentralized organizations and SEs. The internet and ecommerce became a tool for new business models in the late 1990s and early 2000s where people could exchange information and goods and services with each other much easier than before. These $\mathrm{p} 2 \mathrm{p}$ internet platforms allowed people to share assets between each other, both reducing the price compared to the same offering from existing businesses, and offering a new way to make money in the depressed economy. Internet mediated peer exchange included travel, freelancing for projects, merchandise trade, renting assets, trading currency, education, and even lending. These sharing and trading platforms decentralized the supply side of the business model, but usually had a revenue cut being sent to the founders who built and maintained the platform. During the five year period from 2008-2013, decentralized networks with no profit seeking parent organization were also developing. Some examples include Sensorica, Fairmondo, Cocoon Projects, Enspiral, MakeSense, and OuiShare (Manzanedo and Trepat 2017). These networks bring people together around a common, agreed upon governance structure mediated or tracked using the internet or software. All of these organizations not only experiment with technology-mediated decentralized models (modernizing the cooperative model), but are also SEs. While one of Sensorica's goals is to empower 'communities to optimize interactions with our physical environment and realize our full human potential' (Bauwens and Niaros 2016), Fairmondo offers only ethical and sustainably sourced products, Cocoon Projects offers innovation services that improve life quality and experience, Enspiral brings together freelancers working on socially conscious projects, MakeSense is a crowdsourcing platform for helping social entrepreneurs, and OuiShare is working to grow the collaborative economy. These are the first examples of decentralized models coming together with SE to form a new economic structure that we refer to as a decentralized socioeconomic model. Decentralized technology innovation, primarily Blockchain, is now another force bringing the two together, forming a near-majority sector within the \$1Tn decentralized technology market. Blockchain makes new functions technologically possible, expanding the potential to decentralize and scale the equitable nature of cooperatives. Some of these new functions, made possible by putting power into the technology and removing it as a temptation 
to people, include collaboratively defining governance and the definition of value, enforcing business rules, and stewarding the flow of value.

The first network to emerge using decentralized technology with organizational governance was Bitcoin in 2009. Since then, Ethereum was launched in 2015, and over 1300 additional decentralized projects exist with over 600 of these focused on solving a social problem, as previously mentioned (Startup Tracker 2018). These types of technology-mediated decentralized socioeconomic models are the topic of this paper. They are networks that use self-defined value rather than fiat for exchange. By allowing members to define which actions and assets are considered to be valuable, exchange is related to both social and economic factors. However, because the value is technology-mediated, it is different from fiat currency like the US dollar or loyalty points from an airline. If the United States government declared that no more dollars would be printed, United States citizens would have to accept the fact, or influence the government otherwise, and airlines have discretion to nullify loyalty points at any time. By contrast, in these decentralized ledger technology platforms, members are in control through democratic governance consensus processes. There are only a few examples of these decentralized networks emerging where the organizational power, rewards, and definition of value are all defined. Some examples include Backfeed (Backfeed I Spreading Consensus n.d.), Colony.io (Colony n.d.), district0x (District0x n.d.) and Peerism (Peerism n.d.). These models are all fewer than five years old, and are just beginning to uncover the dynamics between the market created by their decentralized socioeconomic model, and existing competitive marketplaces. The history given above is summarized in Figure 1, below.

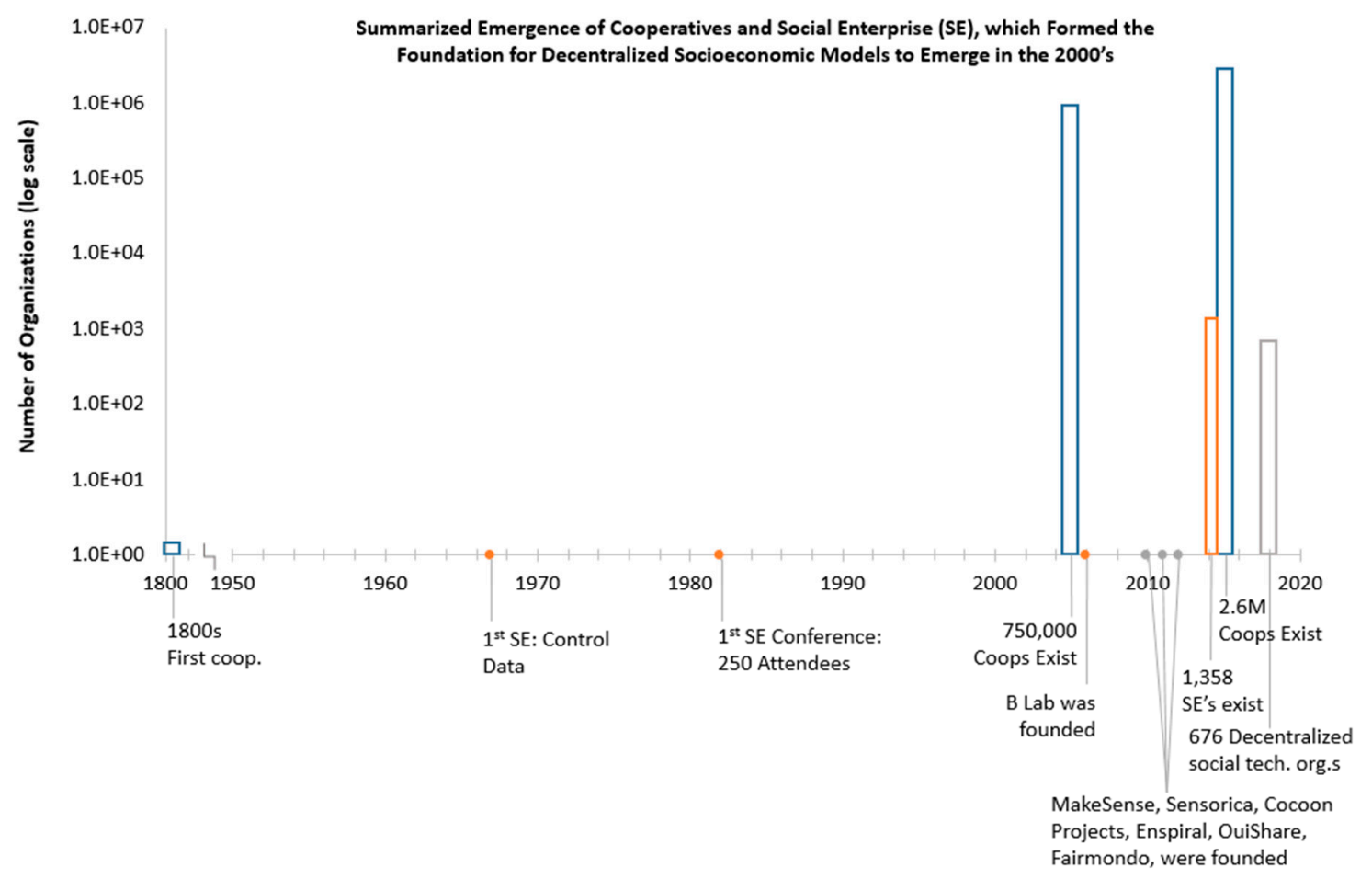

Figure 1. Cooperatives were first recorded near the early 1800s, followed by the first example of the social enterprise (SE) in 1968. Egalitarian control and ownership from the cooperative and purposeful profit from the SE are both embodied in the emerging decentralized socioeconomic models from the past ten years. Notably, the Blockchain based decentralized socioeconomic models like Backfeed, Colony, district0x, and Peerism are not included because the platforms are still under development and testing, and have not reached production scale.

We are specifically excluding models such as R3 CEV (r3.com n.d.), B3i (B3i n.d.) and other private decentralized ledger technology platforms because they are not 
- Open

- Decentralized $^{3}$

- Censorship resistant

- Consensus driven ${ }^{4}$

- Non-competitive $^{5}$

which are key characteristics of decentralized economies.

\section{Decentralized Socioeconomic vs. Competitive Markets}

Decentralized socioeconomic markets and competitive markets are already coexisting, shown in Figure 1 with a cluster of organizations founded around 2010. Through the market comparison and subsequent behavioral discussion analysis below, we will discuss why competitive organizations would be expected to pose a systematic threat to decentralized socioeconomic markets.

If the characteristics of competitive organizations endanger the success of the newer decentralized socioeconomic models, we would only expect three outcomes. One, decentralized socioeconomic models become the new standard and competitive ones disappear, two, we design organizations within these new markets that can coexist with the natural tendencies of competitive ones, or three, decentralized economies either disappear or, at least, never gain any further prominence than now. If we assume decentralized socioeconomic models will proliferate, then the second option seems most realistic, and would be a necessary step to get to one, making it the critical situation to consider. We, therefore, start by explaining a general construct for building resilient decentralized socioeconomic models, then later offer four additional proposals specifically related to developing market interfaces and also internal market operations, which will allow decentralized socioeconomic markets to safely interact and coexist with competitive ones.

\subsection{Decentralized Socioeconomic Markets Definition and Description}

Based on available research, decentralized socioeconomic models, also often referred to as a Decentralized Autonomous Organization (DAO) or Commons ${ }^{6}$ structure typically have three main components (Giotitsas and Ramos 2017; Filippi et al. 2007):

1. Entrepreneurial Common (EC): An EC is the commercial interface with external ecosystems and gives funds it raised from selling goods and services or other activities such as investments in other ecosystems in the form of tokens to the For-Benefit Common (FBC) and receives goods \& services to market and sell from the Production Common (PC) in return. This requires exchange between an EC token and Fiat and a PC token that is governed by the FBC. Tokens generally represent a unit of value as defined by the participants of a DAO, and there may be many tokens within a DAO. In addition, the EC is responsible for financial and monetary policy in the DAO since issuing a token is effectively creating a currency with all the accompanying complexities. We will discuss this in more detail when we discuss our proposals for the coexistence of decentralized economies and competitive markets;

2. Production Common (PC): A p2p group that produces goods and services collaboratively based on the purpose of the ecosystem as established in the FBC. A participant's contributions are valued in PC tokens which can be exchanged to EC tokens or other tokens through an exchange

\footnotetext{
With decentralization we refer to governance, ownership and control as well as infrastructure. Without a Nothing-at-Stake problem and without either federation or lack of imperative mandate.

5 Non-competitive in this context means that participants are not competing against one another or other external entities per se but are aligned to create value around a purpose that is addressing a set of societal or common individual challenges or opportunities.

6 The Digital Library of the Commons defines "commons" as "a general term for shared resources in which each stakeholder has an equal interest" (Digital Library of the Commons n.d.).
} 
utility, as detailed out in one of our four proposals below. Assets created in this common are held in common by the FBC with claims rights by the contributors based on their value contribution to the asset in order to enable a fair sharing of value generated both commercially and through reputation;

3. For-Benefit Common (FBC): The FBC is the governance common that is responsible for setting the DAO vision and impact goals, sets consensus rules and incentives for the DAO commons, sets the exchange rules for the EC and PC Tokens within the commons and externally to other ecosystem tokens and fiat, sets the ownership/membership and sharing rules for the DAO commons, defines and enforces reputation also in relation to non-DAO reputation measurement and management models, sets collaboration and giving rules with internal and external entities, and acts as the interface to not-for-benefit entities etc.

This three-zone model is designed to

- Insulate the economically vulnerable FBC and PC from extractive external markets through the EC commons by limiting token exchanges between the common markets that have direct interfaces to competitive markets.

- Enable social impact results through the FBC without a strong dependency on market results. The FBC decides the use of funds coming from the EC. As a result it is independent of "shareholder value" as defined by external and extractive markets; therefore it is accountable to the PC and EC participants.

- Allows the EC to focus on raising funds for both the FBC and PC either through selling products and services or raising funds for future products and services and social impact efforts.

- Enables the PC to focus on core competencies to create new products and services aligned with the overall DAO values independent of the EC.

It is worth noting that decentralized economies as described above typically have three characteristics in common (Commons Transition Primer 2018, Commons-Based Peer Production Directory 2018, Giotitsas and Ramos 2017):

1. Open Value Accounting: An accounting system that allows one to record not only tangible assets and assess their value in a unit of value measure, but also to record tangible and intangible value contributions from participants to an asset and subsequent value translation into a unit of value measure such as a token in a decentralized socioeconomic model;

2. Decentralized Commons Market: A decentralized marketplace for the free exchange of assets and services governed by business rules established by a governing commons through participant consensus. The decentralized marketplace is transparent and has verifiable and marketplace transactions. In order to motivate participation in a decentralized commons market (DCM), a DCM has an incentive model for both tangible and intangible value contributions and open asset ownership representation through a set of defined unit of value measures such as tokens. The rules for DAO membership and voting are normally based on consensus processes;

3. DCM Reciprocity: Reciprocity in this context means that the return on investment beyond a certain value ${ }^{7}$ is capped but not frozen by requiring reciprocity contributions to the DCM in the form of tangible or intangible value contributions that equal or exceed returns. Example: For a return of a 100 token investment in a DCM asset beyond say 10 tokens, an actor needs to make a value contribution after applying an exchange rate of token to utility token quantifiably equivalent to 1 utility or 1 reputation token for every token earned beyond 10 tokens.

As we will see below, this is in stark contrast to competitive markets.

$7 \quad$ As defined by a DCM governing DAO through participant consensus. 
Central to making the three zoned model operate is effective governance. There is an evolving literature on the governance of decentralized markets discussing issues and challenges creating inefficiencies and potentially additional costs as well as benefits and efficiencies. We will briefly discuss some recent findings but not go into extensive details on governance as this is beyond the scope of this paper or not directly applicable. For the interested reader we mention that (Freund 2018) has extensive discussion on Blockchain supported DAO governance and its processes based on recent behavioral psychology findings and game theoretic considerations around incentives and consensus algorithms:

- Cong et al. (Cong and He 2018) argues that Blockchains enlarge the competitive contracting space while at the same time the process of reaching decentralized consensus changes the information environment enabling "higher social welfare and consumer surplus through enhanced entry and competition, yet it may also lead to greater collusion." Because "Blockchain and smart contracts can sustain market equilibria with a larger range of economic outcomes." This aligns with our above discussion on open value accounting and DCMs with reciprocity and the related discussion in (Freund 2018).

- Yermack points out in (Yermack 2016) that the lack of clear governance principles and rules for decentralized economies such as Bitcoin and Ethereum lead to either inaction in the case of the Bitcoin scaling debate or to ecosystem hard forks in the case of Ethereum. We will discuss in the last section that choosing a hard fork at the protocol i.e., the Blockchain level-is actually a preferable option if consensus cannot be reached or an ecosystem is attacked by a majority collusion attack.

- Cong et al. formally show and provide empirical evidence in (Cong et al. 2018) that mining pools have a natural tendency to centralize because of risk-sharing advantages. They also provide a new explanation for why Bitcoin mining may be adequately decentralized over time due to the higher fee structure of large pools. The paper does not consider collusion scenarios of mining pools to manipulate markets, which is currently a possibility given the concentration of mining power in a few large mining pools. Note that this discussion is only applicable to a competitive decentralized market that is the nature of Proof-of-Work (PoW) or simplistic Proof-of-Stake (PoS). In more sophisticated versions of Proof-of-Stake such as Casper FFG (Buterin and Griffith 2017), rewards are shared, the market is less competitive and decentralization is encouraged through dynamic ROI based on the number and stake of participants changing the underlying market dynamics.

- Easley et al. show in (Easley et al. 2017) that in the absence of transaction fees, over time the Bitcoin Blockchain, in fact any Blockchain, is not viable. However, even with transactions fees, the authors show that there is an upper bound on the size of the Blockchain imposed by the waiting time confronting users in the transaction pool. Users exit the Blockchain if the waiting time gets too long and the costs of waiting significantly diminish the expected gains from a transaction in much the way that miners exit the Blockchain when their revenues no longer generate profits. Hence, the equilibrium in the bitcoin Blockchain is a complex balancing of user and miner participation. Albeit very important, the analysis is only pertinent for PoW Blockchains, because the queueing problem is significantly less in PoS Blockchains, especially when scaling improvements are taken into account. However, unless transaction ordering and transaction censoring are addressed, as we will discuss below in Proposal \#1, the queueing problem may arise again in front running scenarios such as decentralized exchanges. We will touch upon this in Proposal \#4.

- Huberman et al. show in (Huberman et al. 2017) a relationship between congestion and transaction fees in the Bitcoin Blockchain system, which matches network data. It is also demonstrated that, as block rewards are reduced, congestion is essential for raising revenue from users to fund miners' provision of infrastructure. The authors correctly highlight key economic challenges in the design of the markets that underlie these distributed systems though they leave out some key considerations such as non-homogenous distribution of mining power, which makes it more likely for users or their intermediaries to send transactions to large mining pools irrespective of fees. What the paper highlights well is the fact that relying on transaction fees alone is not 
a viable strategy because today's users expect fast response times and low fees of computer systems. This means that new protocols need not only have a dynamic fee schedule but also a dynamic reward and economic stake function that balances validator/miner ROI against the level of decentralization providing system security as is done in Casper FFG (Buterin and Griffith 2017). In addition, adoption is important since higher usage together with available higher capacity in non-PoW systems will allow significant revenue to be created from transaction fees alone, even if they are small.

- Dupont discusses the failed DAO experiment in 2017 (DuPont 2017) and found three key themes of governance related to the DAO experiment that are in line with the discussions on governance in (Freund 2018) and are reflected in our proposals in Section 6, where applicable:

1. shift of legal authority from existing, judicial authority to algorithmic authority;

2. the difficulty of designing and governing algorithmic systems, and especially immutable and decentralized ones;

3. the challenging ethical questions of experimentation with forms of distributed action through autonomous, decentralized systems.

The legal and regulatory questions arising around decentralized economies need to be considered such that people have enough confidence in their legitimacy to adopt them (Poon 2011). Some considerations include:

- What are the legal forms that DAOs can take?

- Do DAOs have to be incorporated?

- How do you establish legal recourse?

- How is ownership established, if at all?

- When are tokens securities vs. currencies vs. commodities vs. ...?

- How can DAOs account for different legal and regulatory boundary conditions in different jurisdictions while maintaining, potentially global rules and processes?

and they have very significant effects on the operations of a DAO.

In the following, we will briefly touch on some of the most important ones without claim to legal authority and given the different meaning of legal terms such as legal entity, recourse, ownership, guardianship etc., we will focus on more general legal characteristics ${ }^{8}$. A good introduction and framework can be found in (Mueller 2018):

- Legal Forms and Incorporation: Entity to entity business relationships are governed by jurisdictional specific laws and regulations. Generally speaking, a DAO that wishes to interact or do business with a traditional company will require a legal, non-person entity ${ }^{9}$ to be established in the jurisdiction in question, otherwise the traditional business will not do business with the $\mathrm{DAO}$, at least for higher value or higher risk business transactions. The legal entity might take the form of a non-profit or for-profit entity and it might or might not exploit particulars of applicable jurisdictional law as for example cleverly done so in (Sundell 2017). The same holds true for intra $\mathrm{DAO}$ value exchanges and transactions if legal, non-person entities are DAO members. However, value exchanges within a DAO or between DAOs (excluding any of their legal entities) do not require legal, non-person entities and are perfectly in line with jurisdictional law as long as those transactions are done between legal persons and adhere to the jurisdictional laws governing legal persons;

8 The comments below were informed by multiple discussions with regulators, and several securities attorneys having done multiple ICOs each and the reading of 60+ ICO whitepapers.

9 Note that the legal definition of a legal, person or legal, non-person entity can vary wildly by jurisdiction. We are discussing the most common form that treats legally registered businesses different from individual people. 
- Legal Recourse: Legal recourse is one of the most important tenets in the current centralized economic model with the current, centralized legal system taking on the role as final arbiter. However, with decentralized economic models, there is typically a priori no centralized arbiter function, but rather a decentralized one based on consensus. In fact, this is not too dissimilar to a jury system except that the legal constructs wrapped around a jury are centralized structures. Therefore, legal recourse in a decentralized economic model takes on an even larger role given that there might not be a legal entity to sue or if there is, they might not hold any liabilities to a product or service in certain cases as opens source software under the Apache 2.0 license. That means, if an event occurs that causes a socioeconomic imbalance in the DAO, there have to be well defined rights of DAO participants to the DAO's underlying assets in all three of its components. Note, that a participant might have recourse rights against one, two, or all three of the DAO structures. Those are rights that should be enforceable programmatically, if certain predefined conditions are verifiably met. This is important since the exact expression of legal recourse varies by jurisdiction, yielding sole reliance on court systems too slow, inefficient, and open to manipulation to be useful for a DAO. The exact details of such an internal legal recourse as well as of external legal recourse are based on the jurisdictions a DAO operates in, and are determined by the governance common, the FBC, through consensus processes. Consensus proposals need to be agreed to by the PC and EC through commons specific consensus processes, as there cannot be unilateral action in this construct. Given the potential scale of a DAO, consensus and incentives take on great importance given the legal recourse requirements. Therefore, a careful definition of the rights and warrants of participants to the underlying tokens representing asset rights for each of the three commons of the DAO becomes imperative and independent if these assets are digital or analog. In addition, there needs to be a careful definition of cascading rights. To make this very important point more concrete, let us consider the following situation: Actor A holds EC tokens that were acquired through an exchange functionality in an environment for fiat currency as discussed in Proposal \#4 below in detail. These EC tokens are subsequently exchanged with the PC for PC tokens through an exchange functionality in order for the PC to create a new good or service. The PC token represents an ownership right. The important question that needs to be answered is what type of ownership this token represents. It could be ownership of the underlying asset or the cash flow associated with that asset. If assets are to be freely used and shared throughout the PC, then the natural answer would be cash flow. If that is not the case, the answer probably would be an asset. Whatever the case, each product and service requires such a determination of ownership rights for contributors both financially and from an asset contribution point of view. This means that the DAO needs to create programmatic business logic representing the ownership rights decisions such that Actor A knows exactly what will happen to his contribution to the PC for a service or product, if a socioeconomic imbalance or dispute occurs. This example is relatively simple, however, for each contributor relationship within the ecosystem that the DAO spans, it is important to have this clarity of cascading legal recourse. A complex but very important task to define, agree upon by participants, and subsequently transform into computer code.

\section{- Ownership \& Control:}

Ownership: When analyzed through the lens of a decentralized socioeconomic context we find two forms of ownership in decentralized economies:

- Self-Sovereign Ownership where ownership rights over an asset are entirely self-sovereign; i.e., "even if shared with others I can do with my ownership share as I please";

- Non-dominium ownership where assets are held in common within a DAO as a custodian and the rights governed by asset rules established by the DAO through consensus. 
An interesting question in this regard is revoking ownership rights. In a decentralized economy revoking ownership rights should not even be possible. The question then is how can DAO entities that abuse ownership rights be effectively censured in order to ensure continued trust into the long term viability of a DAO? A possible answer is to not allow an entity to exercise their ownership rights. For example, leveraging publicly available lists, DAOs disallow interacting with blacklisted assets of DAO participants or the participants themselves. Other DAO participants ignoring such blacklisting can be equally censured since the asset can be tracked through functionality, provided transparency is established by using Blockchain technology. Of course, this discussion depends on courts recognizing Blockchain secured ownership of assets, at least if these assets are utilized outside a DAO.

$\bigcirc \quad$ Control: In contrast to ownership, control of an asset or an actor (guardianship) can be revoked (transparently on a Blockchain) through the owners of an asset or an actor him or herself at any point. Again, the legal discussion reduces to the question of legal sustainability of verifiable claims on a Blockchain in a court of law.

- Tokens: Generally speaking there are three types of tokens:

Native (Utility) Tokens: Tokens representing transferable ecosystem value but do not confer any counterparty rights such as title except what the token holds itself. An example is Bitcoin or any other cryptocurrency ${ }^{10}$ or access tokens to a service;

$\bigcirc \quad$ Counterparty Tokens: Confer a 3rd party counterparty right. For example, tokenized debt to a lender or the right to shares and shareholder rights aka an equity;

O Ownership Tokens: Describes the rights of owners towards an asset such as title. There are no direct or indirect counterparty claim rights associated to such a token.

Given the regulatory inconsistencies globally, within nation states, and between federal regulatory agencies and state jurisdictions, decentralized market projects are starting to gravitate to the most permissive jurisdictions, such as Switzerland, Malta, Cyprus or other known tax havens that are now trying to attract cryptocurrency and token investors. In particular, the Initial Coin Offering (ICO) wave that has raised over \$10Bn for projects since the beginning of 2017 (ICOdata.io 2018) has regulators worried about investor scams, which have already happened at significant scale; for example, a Q1 2018 \$660M ICO pyramid scam in (Coindesk 2018). The challenge for regulators is that on the one hand they want to protect investors, on the other hand they do not want to stifle innovation. However, given the regulatory propensity for caution, and the sometimes conflicting signals that are being sent by for example the US Security and Exchange Commission, uncertainty reigns supreme and furthers the exodus of both money and talent to avoid the risk of being classified as a security. Not only because of the legal ramifications such as having broken securities law but also because security tokens cannot generate the network effects required for decentralized economies, adoption will suffer if you need a broker-dealer license and have to comply with significant regulatory reporting requirements to buy and sell goods and services with security tokens. A recent study into ICOs by Li et al. (Li and Mann 2018) develops a framework to discuss optimal regulation toward ICOs. The authors take an economic perspective, and ask if and when token sales are value-creating or value-destroying from a social welfare perspective. Two specific settings in which ICOs can create value are identified: First, when projects feature network effects - that is, the surplus realized by any user increases in the size of the total user base. Second, when projects feature the "wisdom of the

10 Based on private discussions of the authors with several regulators, a cryptocurrency may be classified as a security, if a predetermined set of people received a set of tokens in exchange for either fiat currency or other crypto currencies/platform tokens with the purpose of financing the building of the cryptocurrency platform before the new cryptocurrency or token platform was launched and/or the issuance was accompanied with an either direct or indirect promise of cryptocurrency/platform token value gain. 
crowd" - that is, private signals about project value that are dispersed among its potential users. It is interesting to see that the multistage launch recommendation of the authors and other governance recommendation aligns with the recommendation on the launch of a DAO, in fact any decentralized network, and identified DAO governance processes made in (Freund 2018).

In Table 1 we give an example of the type of tokens that would be used in a three zone DAO model and how this avoids classification as a security token provided that the tokens were not used to generate financing to build the platform or DAO application layer itself:

Table 1. Delineating token Types and their uses in a DAO.

\begin{tabular}{|c|c|c|c|c|}
\hline Token/Type & Purpose & Usage & Underlying Value & Model Layer \\
\hline $\begin{array}{l}\text { Entrepreneurial } \\
\text { Common } \\
\text { Tokens } \\
\text { (EC Tokens)/Native Utility } \\
\text { Token }\end{array}$ & $\begin{array}{l}\text { Exchangeable for } \\
\text { Protocol Tokens, other } \\
\text { DAO tokens, Fiat }\end{array}$ & Currency & $\begin{array}{l}\text { Fiat, Protocol } \\
\text { Tokens or Assets } \\
\text { held in common by } \\
\text { DAO }\end{array}$ & $\begin{array}{l}\text { Decentralized } \\
\text { Economies } \\
\text { Applications Layer }\end{array}$ \\
\hline $\begin{array}{l}\text { Production } \\
\text { Common } \\
\text { Token } \\
\text { (PC Tokens)/Ownership } \\
\text { Token }\end{array}$ & $\begin{array}{l}\text { Governed by FBC } \\
\text { Values member } \\
\text { contributions } \\
\text { Exchangeable for EC } \\
\text { Tokens }\end{array}$ & Claim Right & Asset backed & $\begin{array}{l}\text { Decentralized } \\
\text { Economies } \\
\text { Applications Layer }\end{array}$ \\
\hline $\begin{array}{l}\text { Production } \\
\text { Common } \\
\text { Token } \\
\text { (PC Tokens)/Native Utility } \\
\text { Token }\end{array}$ & $\begin{array}{l}\text { Governed by FBC } \\
\text { Values member } \\
\text { contributions } \\
\text { Exchangeable for EC } \\
\text { Tokens }\end{array}$ & $\begin{array}{l}\text { Access Right to } \\
\text { Asset }\end{array}$ & Asset backed & $\begin{array}{l}\text { Decentralized } \\
\text { Economies } \\
\text { Applications Layer }\end{array}$ \\
\hline $\begin{array}{l}\text { Platform Token/Native } \\
\text { Utility Token }\end{array}$ & $\begin{array}{l}\text { Participate in platform } \\
\text { and DAO actions. } \\
\text { Confers voting rights } \\
\text { for platform utility } \\
\text { services }\end{array}$ & Currency & Platform Services & Protocol Layer \\
\hline
\end{tabular}

After this more extensive elaboration on the constructs of decentralized socioeconomic models, we move on to the well-known competitive organizations, and the market characteristics of both.

\subsection{Competitive Markets Definition and Description}

Competitive models, by contrast, are assumed to be organizations with some form of shareholder who dictates the expectation of the business's outcomes in exchange for the risk they assumed by investing. ${ }^{11}$ Business outcomes are driven by "value-creating" activities that enhance the competitive advantage of the organization. The organization is assumed to have a centralized governance structure where people in designated power positions define and enforce the rules within the firm. Assets and intellectual property created by employees are owned and held privately by the firm in an effort to maintain a competitive advantage over similar businesses. Finally, we assume that the firm values assets and exchanges in fiat currency.

11 Management of competitive organizations commonly subscribe to the shareholder theory, where their ethical role is to maximize profits for shareholders. In competitive markets today, another management theory is emerging. The stakeholder theory, coined in the mid-1900s and generating more interest by the early 2000s (Arnold 2008), expands the corporation's purpose to serve all entities that contributed to the wealth of the business (Smith 2003). The stakeholder theory, closely related to corporate social responsibility (CSR), expands performance metrics beyond revenue and into the realm of social impact. These impacts are often still competitive in nature, however, and are often only valued by the corporation because the CSR activities actually feed back into a revenue stream (Arnold 2008). A simple example would be an IT company focusing CSR efforts on STEM education programs. No matter which management theory the organization subscribes to, the corporation still remains competitive in nature as it assumes that it will best serve either its stakeholders, or shareholders, by improving its position in the market. 


\subsection{Framework for Market Comparison}

To compare the two markets resulting from decentralized socioeconomic and competitive models, we will use a simple framework of market characteristics:

- Number and size of producing organizations with respect to the market size.

- Barriers to entry of new firms, informed by the ratio of fixed to total cost and network effects.

- Product differentiation and degree of information symmetry ${ }^{12}$.

\subsection{Decentralized Socioeconomic Markets}

The organizational structure for a model that creates a decentralized socioeconomic market has been detailed above in the three zone model. The salient attributes to note are that assets are held openly with no-cost access, and the community defines the model's purpose, means for exchange and governance, and there is a defined border with existing markets. Here are the characteristics of the market:

1. Number and size of producing organizations with respect to the market size:

a. In decentralized markets, there are a large number of producing organizations as the notion of a firm dissolves into project groups within a decentralized network of entities (people, organizations and, in the future, things). This can be viewed as similar to a chemical reactor. The entire model has a mixture of entities and assets interacting with one another governed by adjustable rules. To achieve the model's goal, entities and assets combine in projects, the "producing organizations," to deliver an outcome. Over time, the same entities may frequently work together, but they would not legally constitute a firm, but are rather a commonly occurring set of entities within many projects;

b. The market size is the number of participants potentially impacted by the decentralized model's purpose. We will explain in Proposal \#2 below, the concept of open value accounting, but we can assume that the larger the impact of a decentralized model's purpose, the more members it will have. This is because participants in a decentralized model are compensated in proportion to their contributions when an asset is monetized or has proven impact. Therefore, the larger the purpose, the higher the chance of earning from an asset considering that the network effects of a large decentralized community increases the chance of innovation success and value generated (Torrance and Tomlinson 2009);

c. Since we assume that there is generally a constant optimal project size (Blenko et al. 2010), the ratio of the number of producing organizations per market opportunity is likely to be larger in a decentralized market comprised of short-term project teams than it would be in a competitive market where firms are the producing organization, almost always including more than one project group. ${ }^{13}$

2. Barriers to entry of new producing organizations with respect to market size:

a. The barriers to entry for organizations providing commodities and services are low in these markets because assets are open and free for any member to use and build upon. For products that rely on network effects, like social media platforms or sharing economy services, the barrier to entry will be higher than commodity or service providers, but lower than the barriers to entry of competitive markets where network effects are built on top

12 Information symmetry is achieved when all market participants have the same information available to them at all times.

13 More explicitly, (the number of producing organizations (project teams)/market opportunity) in a decentralized market is greater than (the number of producing organizations (firms)/market opportunity) in a competitive market by virtue of project teams having fewer people than firms because there is no bureaucracy. This is a theory based on logic, not observation. 
of proprietary platforms. Because the products that rely on network effects are open, incremental innovation can be implemented as an update, and come from any member of the decentralized model rather than just the platform founder as in the competitive market scenario with platforms like Facebook and Google;

3. Product differentiation and degree of information symmetry:

a. Decentralized markets operate with globally differentiated products, but locally homogeneous products because of the commons model. This model incents efficient use of existing assets and rewards participants more for added-value rather than recreating existing value. Because members are incented to produce added, rather than recreated value, when an effective solution is in place at the local level, innovators will work to solve new problems related to the decentralized model's purpose. On the global level, however, innovations for the same problem will look different because of contextual innovations that took place at the local level.

b. Because assets are open and transparent from Blockchain-mediation, information symmetry is approached, but not reached due to bounded rationality ${ }^{14}$.

\subsection{Competitive Markets}

Competitive markets ${ }^{15}$ are comprised of many types of organizations, including firms, small private businesses, non-profit organizations, and even individual freelancers offering competitive services on another firm's competitive platform like Upwork. The common assumptions made about organizations that operate within a competitive market are that organizations are profit maximizing, usually have centralized power structures, and gain an advantage by developing differentiated products that consumers prefer. In addition, these are the definitional market characteristics for competition:

1. Number and size of producing organizations with respect to the market size:

- The number of firms is large. This develops competitiveness as each firm works to develop a preferred offering.

2. Barriers to entry of new firms:

- Competitive markets are characterized by easy entry into and exit from the market. Without this being the case, the market would become a monopoly.

3. Product differentiation and degree of information symmetry:

- Products are differentiated, and information asymmetry is a factor that can drive prices since information and assets within a firm are generally closed, and all actions are not Blockchain or technology-mediated exchanges. ${ }^{16}$ Competitive firms can further their purpose of profit maximization by taking advantage of information asymmetry to set higher than market prices that a customer will normally accept in a more information/asset symmetric market; i.e., a high degree of competition.

Table 2 summarizes the two markets below:

14 Bounded rationality means actors make imperfect decisions because of a lack of understanding, time, or access to information.

15 Here we assume imperfect competition to consider real-world, rather than theoretical markets, although we will continue to refer to them simply as competitive markets.

16 Information asymmetry has been reduced by the internet and ecommerce. Easy access to competitors' pricing and quality has reduced this information asymmetry. 
Table 2. Comparison of the primary market characteristics of competitive and decentralized socioeconomic markets. They are similar except for information symmetry, the result of using Blockchain, and product differentiation, the result of collaborative vs competitive incentive models within the market.

\begin{tabular}{ccc}
\hline Primary Market Characteristics & Decentralized Socioeconomic & Competitive \\
\hline $\begin{array}{c}\text { Number of producing } \\
\text { organizations }\end{array}$ & $\begin{array}{c}\text { Many. The producing organization } \\
\text { (project team) is the size of an } \\
\text { effective project team }\end{array}$ & $\begin{array}{c}\text { Many, but fewer because the producing } \\
\text { organization (firm) is usually comprised } \\
\text { of many project teams }\end{array}$ \\
\hline Barriers to Entry & $\begin{array}{c}\text { Almost nonexistent because all } \\
\text { assets are held in common }\end{array}$ & $\begin{array}{c}\text { Low because fixed costs are assumed } \\
\text { to be low }\end{array}$ \\
\hline Product Differentiation & Homogenous locally & Differentiated \\
\hline Information Symmetry & Approaches symmetric & Asymmetric \\
\hline
\end{tabular}

\subsection{Comparison of the Outcomes of These Different Organizational and Market Structures}

The outcome is the difference between monolithic business definition of value in competitive markets and diverse value definitions in decentralized socioeconomic models as tokens or cryptocurrencies. Single-measure and multi-measure value systems have pros and cons. Abstracted, single measures of value simplify market decisions and governance, but at the same time, obscure the meaning of where and how the value was created. This can lead to unintended consequences like the market incenting environmental degradation (Bollier 2017).

One way that decentralized socioeconomic models have avoided being stuck with revenue growth as their organizational driver is by using non-traditional funding mechanisms. The prominent model is an ICO where startup capital is crowdsourced and can grow based on speculation and/or actual value creation from market activity. Without revenue expectations from investors, there is more freedom to define the token value in the FBC, freeing decentralized socioeconomic models from shareholder management.

With these diverse value definitions and access to all assets through the FBC enforced commons rules, a producer earns the most value by contributing to the FBC's purpose and impact goals. This leads to collaborative behavior amongst member participants who collectively compete to find the best solution to external forces like water scarcity, achieving the $\mathrm{FBC}^{\prime}$ 's collective purpose: clean water for all. This means homogenous products are likely to form at the local level-innovators no longer have incentive to recreate solutions with slight differentiation, but differentiation will persist at the global level because unique communities will need different solutions.

This does not mean that competition will not exist within a decentralized socioeconomic model, but the competition will be fundamentally different. Competition will only exist between producing units when innovation is happening simultaneously and a sufficient solution does not yet exist. Any producing unit will therefore compete until a sufficient solution has been created. However, once the sufficient solution has been created, efforts will be directed towards other activities that the FBC deems to be more valuable than the marginal improvements to an existing solution. In competitive markets, marginal improvements earn market share, and advances the organization toward its profit goals. However, the FBC can award value or reputation for actions beyond selling for a profit when they align with the overarching goals, giving producing units a reason to move on from tweaking sufficient solutions for market share, to solving more novel problems. Globally, innovation will remain lively, and productive efficiency will increase because re-creating value is meaningless in an open asset environment. Boldrin and Levine have shown that global innovation increases in a commons model 
when innovation builds on many previous ideas (Boldrin and Levine 2005), while innovation is better motivated through closed intellectual property (IP) when the invention is less complex. ${ }^{17}$

Another implication of diverse value definitions in decentralized socioeconomic models is the ability to accrue value to the members as reputation tokens. These tokens are generated by giving transactions ${ }^{18}$ in the market, and are a key incentive structure for innovation that is not exchangeable. ${ }^{19}$ This has not proliferated in the competitive scenario, likely because reputation as a source of value would require the individual to trust the issuing entity on long time scales. Fiat currency, for example, works because of this type of trust. The American public and international communities trust that the dollar will hold value because of the country's third party ratings of financial trustworthiness and the public-private governed Federal Reserve System of monetary and fiscal policy keeps the value relatively stable. By contrast, if reputation-based value were stewarded by a centralized, competitive organization, the power to change the rules is unchecked. By being governed by the people who run the organization, decentralized socioeconomic models allow reputation to be rewarded to members who give, and the members can trust that it will remain valuable because they are the ones who determine its utility through technologically enforced consensus.

These two models ultimately lead to different expected behavior, although behavior in a global decentralized socioeconomic model is yet to be tested. In the competitive market, employees and investors keep assets and IP closed to win in their market and increase shareholder value. In decentralized socioeconomic markets, members maximize their personal return through asset generating actions and giving actions that are rewarded by the FBC when consistent with purpose and impact goals. This will lead to a plurality of value in global markets ${ }^{20}$ as decentralized socioeconomic models define value in their FBCs, and diversify the global incentive structure of human activity.

\section{Value Extraction vs. Reciprocity: How Competitive Markets and Centralization Endanger Decentralized Economies}

To better understand the reasons why competitive markets endanger decentralized economies, we can consider the main behavioral insight presented in the market comparison section: competitive markets have a single measure of value, while decentralized socioeconomic markets have as many definitions of value as FBCs exist. Because of this, competitive organizations will attempt to interact with decentralized socioeconomic models on the basis of a single type of value, ignoring the importance of nuanced actions that would produce value within one FBC, but not another. Since shareholder value is assumed to be maximized through profit, they will naturally be extractive and seek opportunities afforded by all of the market characteristics of decentralized socioeconomic markets discussed ${ }^{21}$ to convert value from the decentralized socioeconomic model into profit, as if it were a natural resource available for plunder. The following section explains the extraction scenarios, or "attacks" from the decentralized socioeconomic market's point of view, related to each market characteristic.

17 Asset sharing arguably happens within a firm, so the distinction of closed and open IP is the organizational boundary. The competitive corporation's boundaries are limited to employees. The decentralized socioeconomic model's boundaries are members, but unlike a company with limited resources and a limited number of employees, decentralized socioeconomic models can accept an unlimited number of members and scale their protection of the commons with the EC on Blockchain without incurring additional costs.

18 A reputation token represents the appreciation of any justified and finalized giving action without any economic compensation through ecosystem tokens or other ecosystem external assets; e.g., you cannot build a barn and get paid in bread and expect to gain social reputation (Freund 2018).

19 Non-exchangeable value refers to efforts that benefit humankind, but do not produce a product or service that a single person would rationally buy. One example is reducing your personal carbon footprint.

20 Global markets refers to both decentralized and competitive markets.

21 Number of producing organizations, barriers to entry, product differentiation, and information symmetry/asymmetry. 


\subsection{Number of Producing Organizations}

The large number of producing organizations creates attack scenarios where small instances of value extraction multiplied across the large number of producing units poses a profit opportunity. Ways that this could happen would be through an actor encouraging many producing units to assign disproportionate claim rights for superficial contributions via the value equations of an open value accounting system, described in the Sensorica case study and Proposal \#2. This scenario pertains to when the claim rights are defined for contributions to a new asset, or one that is comprised of existing assets. The mitigation for these vulnerabilities are inherent to the three zone model, and outlined in "Model for Consensus controlled, Socioeconomically Incentivized and Platform driven Decentralized Economies and Businesses" (Freund 2018) and summarized below.

The process used to define value equations and calculations prevents value extraction from a competitive actor who contributes to the creation of a new asset. The initial value equation that is developed for a new project (aka producing unit) is generated by defining what is valuable, and the metrics to track that value through different tokens see (Freund 2018) for details. Actors who attempt to choose metrics disproportionately in their favor will either succeed because no other producing unit member notices or feels like it is disproportionate, ${ }^{22}$ or will be punished later by another producing unit member who publishes a malicious action claim against them. Malicious action filing allows punishment of any unforeseen and unspecified malicious actions that are defined, proven, and verified by validators in a staked arbitration process where the malicious person or false accuser lose their stake.

On the other hand, if an actor agrees to a specific value equation and measurement metrics, but then attempts to claim a larger share of value by falsely representing their level of contribution, they will be caught and punished. This is done through the contribution submission process where the amount of value earned is checked using submitted proofs, and participants submitting or verifying provably inaccurate proofs are punished.

The final case is when a competitive actor within a producing unit extracts value by contributing to an asset that is comprised of other, nested assets, but undercuts the other contributors through unfair valuation of their contributions, effectively stealing value from the commons. There are three options available within the FBC DAO to ensure that the value of the nested assets are fairly valued, summarized below and given in more detail in Section 6.2. The first is where the creators of the new asset determine the value to be awarded to nested asset owners. If fairly done, this is efficient, but if unfairly valued, the producing unit who assigned the value is subject to punishment resulting from objections of the nested asset owners. The second option is the same as the first, except the value assessment is voted on by the nested asset owners and updated using an arbitration process where a new valuation is introduced if the valuation is rejected, and the outcome of the arbitration process is final. The final option is for the producing unit to value the contributions, submit that valuation directly to the arbitration process where the nested asset owners propose a different valuation and the outcome is final (Freund 2018).

There are also vulnerabilities if these producing units voluntarily, or by independent economic rationale become more centralized. One example is when a competitive organization adds their asset IP that was created in the competitive market to the decentralized socioeconomic market to gain market share from the members within. In this case, the grandfathered-in IP claim rights would be fully owned by the competitive organization, which is therefore incented to make it leveraged as much as possible. One way to do this would be by creating cartel like conditions where almost, if not all, capable producing units are coerced or naturally centralized to only producing the grandfathered-in asset. Details of the tendency for producing units to centralize are mentioned in Cong et al. within the context

22 The feeling of value being disproportionately earned by an actor is subjective to the producing unit members, but solidified in objective measures through the consensus process. Therefore value earned can only be disproportionate if other producing members feel it is. 
of Bitcoin mining (Cong et al. 2018). Centralization would be likely to occur to achieve economies of scale or spread risk across a pool of contributors. If economies of scale are reached, for example, it would reduce the cost of assets produced using the grandfathered-in IP compared to similar alternatives, giving it market advantage through price in the decentralized socioeconomic market.

When (more) centralized scenarios occur, the competitive organization/actor could attempt to assign a disproportionate amount of value to the grandfathered-in asset, which is mitigated through the process mentioned in the producing units section above. The structure of the commons also naturally mitigates the probability of this type of attack through open value accounting. With open value accounting, the value opportunity of selling within the market is determined by consensus of all contributors, creating equitable value distribution. In the competitive market, competitive organizations (leaders within the firm) determine the value split for the contributors (employees who accept a contract for hire). Therefore, this value extraction scenario is only economically incented when the market size within the decentralized socioeconomic market is large enough to offset the sacrifice of margins as a result of equitable value redistribution.

\subsection{Barriers to Entry \& Product Differentiation}

These two characteristics are combined because the value extraction opportunity is defined by both. Here, it is the opportunity for a competitive organization to sell something within the ecosystem at a lower cost than existing offerings that are in alignment with the value of the FBC. For example, a FBC that values clean water will encourage the production of products without harming water quality, and therefore may have a higher exchange price than products produced without consideration to water quality. Selling these products at a lower cost would destroy value in the form of clean water without punishment from the decentralized socioeconomic market. There are two main ways to protect against this: enforcing open assets and requiring a stake to join the DAO. Both protect against price cutting using products that are undifferentiated from the customer's perspective, but differentiated from the FBC's perspective by their impact to defined values.

By joining the market's DAO, a competitive organization will be required to provide a stake that is used to punish violation of market rules, and to open assets, even IP, that contribute to products sold on the market. This would mean that a competitive organization attempting to undercut existing solutions will incur the cost of losing their IP rights, which confer an advantage within the competitive market, and also risk their stake and incur the opportunity cost of having it escrowed in a DAO. An example of when the stake would be taken is if a member is found to access the open assets, particularly knowledge, and replicate it in the competitive market to generate profit. The stake and open asset requirement will result in a tradeoff between the barriers to entry, which are high for a profit-only seeking entity but low for an entity aligned to the FBC's goals, and the value expected from producing undifferentiated goods at a lower price to buyers within the decentralized socioeconomic market.

\subsection{Information Asymmetry}

Exchanges such as currency exchanges are critical facilities to reduce the counterparty friction of doing business involving different currencies and assets; more generally, involving different asset classes. Centralized exchanges allow for large market makers to emerge, provide liquidity, especially in less liquid asset pairs, and demand fees for this service. In fact, exchanges can become market makers themselves, which can become a problem in the event of a "bank run" when the market demands large liquidity in certain assets because of a perceived value or liquidity threat in another asset. If there are not sufficient liquidity providers, market values will collapse as witnessed most recently during the Global Financial Crisis of 2008/2009.

Furthermore, the motivation of large financial institutions to be involved in asset exchanges is to generate profit by exploiting arbitrage opportunities due to participant asymmetries in market information and market friction such as access to assets and ability to trade them. Since these advantages are often small and short lived due to the highly competitive market space, participants 
exploiting these asymmetries need to deal with large asset amounts and, often, high frequency trading capabilities. Furthermore, exchanges of large asset amounts allow "attacks" on the value of assets. For example, short selling large amounts of an asset such as a currency often leads not only to its devaluation compared to other assets but also increased interest rates for the asset issuer for two reasons. The asset issuer is often perceived as less creditworthy and hedges are often buying bonds or derivatives of the asset. Defending against these attack scenarios requires large asset and fiat currency amounts, information symmetry and quick reactions. Decentralized economies have potentially enough assets but, unless power has been delegated to a small group of representative individuals, to achieve the latter will be difficult. In addition, such a "centralization" of power even in a federated model is not always desirable from a governance perspective as federated democracies such as the US or France demonstrate. Furthermore, the above described types of asset attacks are made based on a pure profit motive, which is value extractive without any value reciprocity. What happens in these instances of value extraction attacks both in economics and in nature is well documented; the collapse of countries, or even entire regions, and natural ecosystems, such as coral reefs where first the corals die because of ocean pollution, then the small cell animals relying on the corals for food die off and so on, all the way to the largest predators such as sharks that need to abandon the reef for other food sources, leaving behind an ecologically dead wasteland. Therefore, measures have to be put in place by decentralized economies in the interfaces with external markets that prevent rapid value extraction and that require reciprocity.

An additional problem for decentralized economies arises because tokens are currencies, either by design or de facto. Therefore, unless the ecosystem is very large and robust, it is highly vulnerable to value extraction attacks using currency arbitrage. While tokens in decentralized economies are coupled to underlying assets such as code or service discounts, fiat currencies are decoupled from underlying asset values such as gold and their value is based on the financial and political stability of the issuing countries, in other words, the financial trust that others place in the stability of a country. An emerging decentralized economy does not carry the same level of financial trust. Hence, attackers can use this trust asymmetry to extract value out of a decentralized economy in the same way as we described above.

\subsection{Other Value Extraction Opportunities}

Another value extraction attack is the misappropriation of an asset that is important for a decentralized ecosystem to function from its originally intended utility. Such an attack is driven out of a desire for profit, and consequently, prevents the ecosystem from fulfilling its intended purpose. A good example is Bitcoin. The original intent of Bitcoin was to be an unrestricted, economical, and decentralized alternative to fiat currency that was not subject to the central control of governments. Although Bitcoin still has a relatively high level of decentralization and anyone with an internet connection can access it, the exponential rise in price has made Bitcoin equivalent to Gold as a store of value rather than a currency with transaction fees orders of magnitudes higher than just a few years ago. This run up is often described as an asset bubble akin to the Internet bubble (Lewis 2014), the Dutch Tulip bubble (Goldgar 2007) or Beanie Babies (Bissonnette 2015) where asset values were driven up without any underlying real value. Only time will tell whether Bitcoin will remain a store of value or not. What can be learned from this example though is that the tendencies of competitive markets to create asset bubbles leading to Lemon Markets (Akerlof 1995) can have detrimental effects, not only for asset investors, but also the underlying ecosystem; see the effect of the Housing Market bubble of the early 2000s on the global economy leading to the Global Financial Crisis of 2008/2009. In the case of Bitcoin two protocol inherent effects exacerbate the situation: The limited number of transactions driving up transaction fees and subsequently price due to a supply (transaction throughput) demand (many people wanting to trade Bitcoins) imbalance and the cap on the number of Bitcoins creating the correct perception that with limited supply, prices will continue to go up if the current demand persists. Both protocol characteristics were intentional. The latter having the economic intent to avoid 
the inflationary scenarios of fiat currencies. What is a strength in certain scenarios is a weakness in this one; the protocol has no way of moderating asset prices and transaction fees, in other words the forces of competitive markets, through a deliberate fiscal and monetary policy as is done by governments through central banks. The takeaway here is that without mechanisms to create and enforce fiscal and monetary policy, decentralized economies will always be vulnerable to these type of misappropriations due to a desire for profit. Setting monetary and fiscal policy is a non-trivial affair. The current approach by governments is through interest rates and money supply. By their very nature that is difficult for decentralized economies to achieve. We will discuss ways fiscal and monetary policies can be set and what other mechanism can be utilized to avoid the above scenarios in the Section 6.

All of the aforementioned value extraction scenarios focus on market characteristics at the application layer of the Blockchain implementation, and assume a functioning Blockchain protocol and consensus mechanism. The final value extraction attack we will consider is a cross-layer attack that happens when value is extracted from that app layer by attacking the protocol layer, which is addressed with Proposal \#1, specifically in the attack vectors and mitigations discussion. A cross-layer attack can happen when the costs of malicious behavior at the protocol layer are smaller than the value to be gained from corrupting the app layer. This applies to any of the above scenarios. A simple example is if a malicious actor successfully mines a fork that becomes the longest chain, but intentionally wrote transactions to himself in the new blocks, stealing that value from the owners who held it in the honest chain. Defenses against such attacks are robust consensus algorithms at the protocol and application layer as discussed in more detail in Proposal \#1.

\section{The Role of Market Interfaces in the Proliferation of Decentralized Socioeconomic Models}

Market interfaces are the designs that protect the decentralized socioeconomic model from value extraction attacks so they have time to mature. Decentralized markets do not seek value extraction from others to ensure their own prosperity, nor do they measure themselves by the amount of value extracted. They measure success based on the FBC's defined measure of value. Because this value approach is new, it is significantly less trusted than established value models like abstracted fiat currencies. Trust in a business model and its value definition are key to financial and commercial viability. This was most recently demonstrated during the Global Financial Crisis of 2008/2009 when trust in business models and value definitions plummeted.

This means that until this new type of value definition and representation is widely trusted and accepted as exchange from third parties, participants in these ecosystems will struggle commercially, making these ecosystems frail as highlighted in the previous section. Therefore, protective policies in the form of well-defined market interfaces are necessary to give them time to mature and to allow the decentralized community to remain value sovereign ${ }^{23}$ as they coexist with competitive markets (Bauwens and Niaros 2016).

When designing these interfaces, the core design goals are network autonomy and value sovereignty in addition to the three zoned model we suggest for the core design of decentralized socioeconomic models. Value sovereignty is preserved through the interface design between the decentralized socioeconomic model and non-member organizations. Based on evidence of enduring decentralized socioeconomic models like Sensorica and Enspiral, the interface design requires two primary components. The first is a system of value flow between new and existing markets, also referred to as transvestment because value is transferred horizontally between

23 Value sovereignty is "the capacity to self-regulate its relations with the market and to assure that significant aspects of its common wealth and social relationships remain inalienable-not for sale via market exchange." Put another way, "... a commons must be able to develop "semi-permeable boundaries" that enable it to safely interact with markets on its own terms. For example, a coastal fishery functioning as a commons may sell some of its fish to markets, but the goals of earning money and maximizing profit cannot be allowed to become so foundational that it crowds out commons governance and respect for ecological limits." (Peer Production License-P2P Foundation n.d.). 
economic paradigms. The second is an accounting system that tracks value within the decentralized socioeconomic market. In the case studies below, we will explore four market interface models that have been developed for decentralized socioeconomic models, analyze their level of maturity in each of the three zones, and point to vulnerabilities as a result of their interface and core designs. Excluding Ecuador's FLOK case study because it was never implemented, the success level of the organizations in the case studies further substantiate the value of a well-developed three zone model. Their designs have been deployed within the existing competitive markets and offer empirical evidence that informs the four proposals in Section 6.

\section{Case Studies in How Decentralized Economies Interact with Competitive Markets}

We will employ the following Case Study Format:

- Introduce the market's purpose.

- Define value flow at the edge, aka: market interactions and accounting within the system.

- Note any important outcomes of the interface design.

- The three zones of our proposed decentralized socioeconomic model design encompass functions of existing organizations, even if they currently are not delineated into three zones. We will discuss the strength, weakness, or absence of the functions of the three commons (EC, FBC, PC) within each case study, and any resulting vulnerabilities to illustrate the importance of well-developed zones.

1. Sensorica is a network of people who self-govern to innovate and design sensors (Bauwens and Niaros 2016).

a. Members of the network, and any other party, have open access to all contributions of other members, allowing them to mix and match, repurpose, and build upon each other's contributions. Innovation happens in two ways. One is in the context of intrinsically motivated innovation, and the other is market-funded projects that they rely on their commons to deliver. Their accounting system, called open value accounting, tracks all members' contributions, allows them to be valued through a value equation that was previously agreed upon and rewards members proportional to those contributions when a product or service is externally monetized. In addition to project contributions, members earn reputation based on quality of work and in recognition of actions aligned to the network's collective interest. This value is used to assign roles in future projects and provides an assessment of the fairness of activities happening across the network to build trust between members.

b. The market interface is their ability to sell products, services and be sponsored by corporations on open source projects. This flows money into their network while giving them independence from external shareholders. This insulates their value sovereignty from extractive forces by avoiding the need for investors and only accepting funding through grants as needed.

c. Up to this point, Sensorica has been able to protect their network from extractive forces when competitive organizations follow initial agreements. However, they have interacted with corporations who do not uphold their initial agreement, and forfeit payment to the network. This highlights a key vulnerability of their server-based infrastructure. They are not able to enforce agreements easily without legal recourse. A Blockchain infrastructure with programmatically executed contracts could automate payment based on defined metrics or milestones, potentially alleviating this weakness. However, Sensorica has developed one of the most successful operating incentive structures that organizes the efforts of decentralized members around the FBC's purposes. Their model was key to informing Proposal \#2. 
d. The FBC of Sensorica is relatively strong. They have defined governance, and a clear, defined purpose with matching incentives through open value accounting. The EC is relatively weak because fundraising is carried out spontaneously by members, or on an urgent basis to fund specific network needs. Projects and products are sold similar to in a small business, but their network's small size and equitable distribution of revenue to contributors means that there are no large stores of value in the network available to fight legal battles when larger corporations violate their contract. The PC is relatively strong, with the assets produced by the members held in a not-for-profit called a custodian and open for all members of the PC to access. In addition, open value accounting creates a defined set of rules and reward expectations that all members agree and operate on, aligning incentives of contributors with the values of openness held in the FBC. The vulnerabilities mainly come from the EC, where funding is weak, limiting the level of mobilization of PC participants to carry out the FBC's goals. The lack of a well-defined EC with reasonably enforceable contracts means that the network is prone to net value loss to external organizations as they take advantage of open assets without reciprocating value.

2. Enspiral is a supportive coalition of and for social entrepreneurs.

a. As SEs, the members of Enspiral operate like competitive businesses but with a social mission. They use fiat currency and traditional accounting to offer products and services. Enspiral LLC acts as the central node of the SE network. Members can contribute to this node's budget, which is democratically governed by participating members to determine how funds are allocated.

b. Enspiral interacts with the competitive market by issuing shares to investors and capping returns to a specified rate in a model referred to as "capped-returns" transvestment. Once a specified return rate is reached, the SE buys back the investor shares and is able to reinvest all future profits into its mission.

c. This model avoids perpetual extractive shareholder interests by raising initial capital from the capitalistic economy, then buying back all shares to become an autonomous business free to act in the interest of their mission, not shareholders.

d. The FBC is strong, and structured as a central node comprised of members who vote on changes using a centralized software tool called Loomio. The limitation of using a centralized decision making tool for all governance and decisions is the nothing at stake problem, opening the network up to human-engineering type attacks like discouragement, extortion, or collusion whose mitigation are chiefly discussed in Proposal \#1. The central node also accepts donations from member organizations and individuals, acting as the EC, which distributes the funds democratically through member vote to projects that serve its purposes. The other component of the EC is the capped returns policy for raising external funds from investors by strictly limiting the amount of return on investment that can be earned and extracted from the network, and prohibits funds (except for wages and covering operational type costs) from being withdrawn from the network so that the maximum amount is reinvested into its purpose (Financial Agreement Enspiral Handbook n.d.). Enspiral's PC is relatively weak, and is simply the combination of SEs that are incented to work together by intrinsic motivation, rather than by design. The control mechanism for selecting members who will be intrinsically aligned is through invite and nomination-only membership from existing members. The weak PC introduces a possibility that members will not maximally contribute to Enspiral's overall benefit because each member organization must succeed on its own. Without strong extrinsic incentives to contribute or formal requirements of open assets, the strength of collaboration within the network relies on intrinsic alignment of contributors (Venture Agreement Enspiral Handbook n.d.). 
3. FairCoop/FairCoin is a group of coops founded on the values of cooperation, ethics, solidarity and north-south redistribution and justice in economic relations (Faircoin-P2P Foundation n.d.). The FairCoin has been developed to become an alternative currency for exchange and a store of value within the FairCoop network.

a. FairCoins are designed to be an alternative currency to fiat. Members can participate in FairCoop market exchanges with them. Once coins gain enough value in the market, they can be used to fund cooperative projects that FairCoop members care about. There is a static coin supply that cannot be changed, even through consensus (König 2016).

b. The market interface is the exchange mechanism between fiat and the coin. This is their means of transvestment, and bootstraps value into the cooperative network. To establish value sovereignty, they developed an incentive mechanism so members are able to use FairCoins as an exchange mechanism and store of value. This is done by rewarding members who hold a minimum number of tokens for a specified time period. This reduces volatility from extractive investors who buy large amounts of tokens, hold them until the price increases, and then exchange them back to fiat without reciprocating any value into the ecosystem.

c. An important distinction here is that value sovereignty is not enforced, but rather encouraged. Since its launch in 2014, its value has increased along with many other cryptocurrencies, and has been successfully used as an exchange mechanism with the FairCoop ecosystem (FairCoop 2017). We consider the permanently static coin supply to be undesirable, although it was done intentionally to increase the coin's ability to store value (König 2016). FairCoin is therefore designed with fewer ways to address value fluctuations from internal market activities, external factors like speculation, or arbitration attacks. The long term stability and success of FairCoin as a means for exchange over time will provide evidence for or against our Proposal \#3.

d. The FairCoin is only a tool of the FairCoop organization, so the discussion around the three zones will focus on FairCoop. The FBC has started with founding, unalterable values, but strategies and other things can be changed (FairCoop Principles n.d.). Changes are made through four councils that propose changes, and must reach $75 \%$ majority vote from participants. The EC is governed by FairFunds, an organization within FairCoop that holds FairCoins from the initial token distribution, and also accepts donations to support new projects that contribute to FairCoop's values. The new projects are governed by a democratic council and only receive funds after milestones are met (FairFunds n.d., Faircoin-P2P Foundation n.d.). Otherwise, the EC also includes policies that FairCoins can be purchased in exchange for fiat at will. The main vulnerabilities are that the FBC defines unalterable values, which may become ineffective in the future, and the inability to alter them may cause misalignment with members and abandoning the ecosystem. The EC is also dependent on the value of the FairCoin to increase and remain stable, as it is the main funding mechanism for network growth. This highlights the need for Proposal \#4, decentralized exchanges, to support the stability and reduce the probability of attacks on the token, which is central to FairCoop's operations. The PC is supported by an ecosystem of common resources like FairCoin and FairFunds. Local nodes are expected to contribute knowledge to the global knowledge commons of the FairCoop, and global knowledge is also expected to flow to local nodes, although it is unclear how this norm is enforced (Faircoin-P2P Foundation n.d.).

4. The Ecuador FLOK (free/libre, open knowledge) Society was a project initiated when Rafael Correa came into power, rewrote Ecuador's constitution, and was determined to link "economic and social life to the values of personal well-being and protection of the environment" (Bauwens and Restakis 2015). Research, planning, and policy proposals were developed to implement a 
FLOK based economy that could give Ecuador independence from extractive western nations that were buying Ecuador's low-value raw materials, and selling Ecuador high-value refined products. This commons model would realign Ecuador's economy to an infinite resource-knowledge, and away from finite natural resources.

a. People contribute to the commons, paid or unpaid. In the transition period before the commons economy becomes dominant, the state will pay commoners for the investment period when a livelihood cannot be made by contributing to the commons. Those people are taxpayers as well, and generate the knowledge, code, and resources that go into the open commons. The commons are managed by non-profit foundations. These foundations ensure the open licenses on commons assets are not breached, and raise money for infrastructure. Their work also gets contributed to the commons. People who contribute to entrepreneurial projects, and who contributed to the production of the assets held in common that were used, are compensated in a commons-measure of value. This is the internal value regime, independent of the existing competitive market. Because these "ethical companies" will be opening their IP, they will be less attractive to investors looking for profit maximization. To support these mission-oriented organizations, alternative economic systems for them to operate on must be developed and politically supported.

b. Businesses and entrepreneurs are able to access the resources in the commons, employ people to help add value to the common assets, and provide funding for commons infrastructure. The surplus social value accrues back to the commons because a business that privatizes knowledge from the commons is required to pay for that asset used, as stipulated by the peer production license of commons-based knowledge.

c. This model was developed and several policy recommendations were made, but the Ecuadorian government did not implement all of the policies. To date, the implementation of these proposals includes the mandate for all academic texts to be made open, and the beginning of a transition of government software to free and open versions (Brown 2017). Because of this partial transition to a commons based economy, we cannot determine the framework's success.

d. The fact that this case was never fully implemented is the major consideration. The FBC is government controlled, and never was implemented. If it had been, this centralized control would subject the community to unilateral changes in the market, introducing unpredictability, and therefore lack of trust as a long term, viable market to base livelihoods on. The instability of central control is another motivation for Proposal \#1.

\section{Protection \& Coexistence: A Working Proposal to Protect Decentralized Economies}

After having set the stage in the previous sections, in this last section we will discuss ways decentralized socioeconomic structures can protect themselves from and coexist with competitive markets. Our proposals are in parts both alternative and complementary to existing proposals, such as in (Bauwens and Niaros 2016), and are focused on providing scale, security, and robustness to the three zone model of a DAO (FBC, PC, and EC) and its three key characteristics of open value accounting, decentralized common markets (DCMs), and DCM reciprocity that we detailed in Section 3.1 by mitigating the challenges and potential attack vectors discussed in Section 4 . These four proposals should be taken together, but could also be implemented separately, depending on the situation at hand. They are:

1. Collusion Resistant and Tamper Proof Consensus Governance to avoid the illustrated value extraction attacks at both the platform and application layer. This proposal is also foundational for the other proposals to be really secure; 
2. Programmatic Value Recourse: Equitable Asset Participation that establishes built-in value reciprocity limiting value extraction either unintentionally or maliciously through collusion attacks;

3. Semi-Programmatic Monetary and Fiscal Token Policy to avoid liquidity value extractions attacks and enable to enhance network effects as a secondary benefit when certain fiscal and/or monetary conditions in the DAO are met;

4. Decentralized Exchanges to prevent value extraction through arbitrage stemming from information asymmetry and access restrictions to trading facilities and other means by requiring trading collateral and trading stakes.

Given that platforms such as Ethereum have both protocol and application layers that are economically relevant, we will refer to both application and protocol layers within the proposals where required.

\subsection{Proposal 1: Collusion Resistant and Tamper Proof Consensus Governance}

To achieve high-levels of decentralization and operability, which creates security through trust and scale, economies and business models require a process by which a group of participants can reach a decision on a question. This is called decentralized consensus. Decentralized consensus needs to be both practical, efficient, and highly secure and, therefore, trusted. This means that a consensus process needs to be as collusion resistant and tamper proof, and therefore, secure as possible. Our base assumption for an ecosystem is that even if actors "think" they can trust one another, which we call qualitative trust, they have no way of being able to derive a quantifiable level of trust within an ecosystem. Therefore, we need a consensus process that achieves that. The Bitcoin Blockchain was the first to achieve this type of quantifiable trust through economically incentivized consensus, effectively marrying behavioral economics and software at the protocol level. However, this was at the expense of efficiency and to an extent practicality due to its use of the very compute intensive PoW consensus algorithm. Given this constraint, we need new types of socioeconomically incentivized consensus process(es) that will yield the above requirements for practicality, efficiency, and trust. Below we summarize our consensus recommendations as a set of characteristics:

1. All consensus processes should utilize a proof-of-(resource)-stake algorithm ${ }^{24}$ utilizing Blockchain tokens defined at both the protocol layer, such as Bitcoin, and at the application layer, such as an ICO token. Proof-of-(resource)-stake is recommended to avoid the energy waste of PoW and making consensus processes more easily implementable and scalable across a large participant group without significant compute requirements;

2. Tokens are earned through rewards (new tokens) and fees charged by participants that provide utility services to a DAO. Minimal fees should be defined in a schedule per utility service. The fee schedule is governed by consensus of all actors participating in utility services and should be dynamic. A Utility Service is a consensus governed service such as Block validation provided by a set of staked ecosystem participants running a consensus algorithm either at the protocol or application layer;

3. Participants can pool tokens to become a staked validator in a consensus protocol: One validation node with multiple pooled stakeholders is, therefore, possible;

4. Proof-of-(Resource)-Stake consensus algorithms should have a mathematical proof of security;

5. The nominal token value of the reward for a unit of a utility service, such as a Block, is determined through consensus of the ecosystem initiating actors and is recorded within a Blockchain.

24 Proof-of-Stake = An Algorithm that requires participants to provide an economically meaningful stake of some type that allows them to participate as a network validator in a consensus protocol and earn money through rewards. The economic stake can be lost if a validator violates specific rules of a consensus protocol. Therefore, the economic stake acts as a deterrent to malicious validator behavior. 
Subsequent changes require a p-majority agreement of ecosystem participants where $p>50 \%$. The reward should be dynamic such that it maintains a rate of return as has been agreed upon by the utility service participants through consensus, irrespective of the number of participants in a given consensus round;

6. Non-utility services including governance processes requiring external validation should follow a simplified model of staked validators etc., as described in Model Assumption 16 in (Freund 2018).

The ability to adjust rewards and fee schedules after launch is an important requirement for an ecosystem to set its own fiscal and monetary policy by either enhancing or reducing incentives and token production rate.

Saleh shows in (Saleh 2017) that even a simple PoS algorithm will reach consensus at all times if the protocol enforces a Longest Chain Rule ${ }^{25}$ and, therefore, can be deemed "safe". (Pass and Shi 2016a, Buterin and Griffith 2017) propose much more elaborate PoS algorithms with much stronger security, liveness and fairness guarantees. Counterarguments about the danger of centralization in pools as discussed in (Cong et al. 2018) are given, but can be mitigated through dynamic reward and fee schedules as they will be applied to Casper FFG in order to encourage decentralization by reducing $\mathrm{ROI}$ if stake concentration in the committee is detected. Another counterargument supported by the work of (Easley et al. 2017) around diminishing revenues if users abandon a chain due to long wait times, is not applicable for PoS chains since the queueing game assumptions applicable to Bitcoin are not applicable in a PoS settings with blockrates of $1-5 \mathrm{~s}$ as possible with today's algorithms.

\subsubsection{Attack Vectors \& Mitigations}

Before discussing the safety of our recommended consensus protocols, we need to stress that all of the below is predicated on the security of the underlying cryptographic primitives used in those consensus algorithms such as digital signature schemes, hash functions, and cryptographic commitment schemes. We recommend only to use battle tested approaches such as Elliptic Curve Cryptography used in Bitcoin and Ethereum or other public Blockchains.

Consensus algorithms have different vulnerabilities depending on consensus type and the employed economic incentive models. For example, we know that PoW is vulnerable to a 51\%-attack of combined mining power that would allow an attacker to rewrite Blockchain history. A good overview of current consensus models at the protocol level and their security levels is given in (Bano et al. 2017).

In the above class of recommended consensus algorithms at the protocol level, we heavily rely on the work in (Pass and Shi 2016b, Buterin and Griffith 2017) that discusses both mathematical and economic proofs of security for particular PoS algorithms, namely Snow White and Casper FFG. We will not go into the mathematical details but rather highlight the type of attacks and how they are mitigated:

1. Collusion Attacks: A 50\%+ stake majority of consensus participants collude to alter Blockchain history. Mitigations: Stake is not equivalent to voting power but rather gives a participant a higher chance to be part of the voting committee, otherwise it is one member one vote, making collusion more difficult, especially in larger committees. Furthermore, the consensus algorithms we propose are probabilistic, meaning that at any given point in time there are several active chains but only one of those will turn out to be the longest chain. This means that if a collusion attack is detected, the minority can abandon the corrupted chain and continue with a chain that they trust. If economic finalization of an epoch is colluded against, the same approach of chain switching can be taken. In addition, committee membership can change and/or be different on another chain and that committee can gain enough votes for finalization. At that point, the colluding

25 One fork that is k blocks longer than any other fork, where $\mathrm{k}$ is either dynamically determined or static, and is determined to have achieved consensus. 
participants need to switch to the new longest chain with the now finalized checkpoints or risk losing all their stake due to a violation of protocol rules that disallow conflicting checkpoints with a majority of votes;

2. Long Range (Revision) Attacks (Nothing-at-Stake): A 50\%+ majority of Committee members who voted on a checkpoint in the past but have now since unbonded their stake could vote on finalizing a conflicting check point after the one they last voted on, creating an alternate chain. Mitigations: Long unbonding periods in the protocol make these attacks more difficult because the other participants will have seen a lot of other checkpoints finalized and will not accept a conflicting checkpoint at a lower height. There is the issue of a large number of participants having been offline for a longer period of time. This would be a potential problem, however, the protocol's liveness slashing condition ${ }^{26}$ mitigates this type of attack;

3. Discouragement Attacks: Examples of such attacks are censoring transactions or not participating in consensus in order to prevent checkpoints from being finalized. Mitigations: The latter type of attack is mitigated by the protocol's liveness stake, slashing conditions, and the former attack can be mitigated through the staked consensus of the trusted hardware overlay networks (Bakshi and Freund 2018);

4. Extortion Attacks: These type of attacks are a variant of censorship attacks where the attackers either refuse to process transactions or vote on check points unless participants pay an extra fee or collude with them in one of the above three other attacks. Mitigations: As discussed under Discouragement Attacks, censoring actions can be mitigated by trusted hardware overlay networks and the former type of extortion attack can be submitted as a malicious action transaction and can lead, if approved by a majority of the committee;

5. Committee Manipulation Attacks: This is a very complex subject matter dealing with the selection algorithm, its inherent entropy etc., and potentially quite dangerous since it allows the manipulation of the chain by a malicious committee (see collusion attacks). Mitigations: We refer the reader to the details of the Snow White and Casper papers we cite. The bottom line is that given the chosen constructs, manipulation of committee selection is becoming extremely difficult;

6. Participants synching to a malicious chain after being offline for a long time: Again, this is a very complex subject matter dealing with different types of sophisticated attackers and quite dangerous since it allows a malicious chain to persist unbeknownst to honest participants. Mitigations: We refer to the details of the Snow White and Casper. The bottom line is that given the chosen constructs, manipulating synching participants is becoming extremely difficult.

Besides the consensus algorithms at the protocol level, we have to discuss consensus algorithms used at the application level to manage DAO processes and interfaces through consensus on the state of events or business outcomes. Since we have varying types of states that require consensus compared to the protocol level, we can relax some of the assumptions of Snow White and Casper, such as not voting on conflicting checkpoints because that case cannot happen at the application layer due to the fact that the underlying consensus protocol ensures the security of the Blockchain state upon which a DAO builds its business state. We can, therefore, use a simplified version of their PoS consensus algorithm utilizing blinded committee selection, cryptographic commitments, simplified slashing conditions, and the possibility to challenge a consensus if it appears to have been the result of malicious action as detailed in (Freund 2018) and also as part of Proposal \#3 below while maintaining strong security guarantees.

The attacks for which this consensus algorithm is susceptible to are the same as at the protocol level. We will, therefore, just discuss the mitigations of the attacks in this consensus protocol:

26 If validators do not participate in voting for a certain period of time they lose all of their stake over time. 
1. Collusion Attacks: Mitigations: Stake is not equivalent to voting power but rather gives a participant a higher chance to be part of the voting committee, otherwise it is one member one vote, making collusion more difficult, especially in larger committees. If vote finalization within a voting period is colluded against, non-voters lose their stake and a new committee is selected. Furthermore, if there is a suspicion of collusion, anyone can request a revote if the request is staked (skin-in-the-game) with a new committee further reducing the possibility of a successful collusion attack;

2. Long Range (Revision) Attacks (Nothing-at-Stake): Mitigations: Not required since the underlying consensus algorithm protects against this type of attack;

3. Discouragement Attacks: Mitigations: Censoring a vote is not possible if submitted to multiple Blockchain nodes at the same time and non-voting leads to a loss of stake as discussed before;

4. Extortion Attacks: Mitigations in this case are the same as for discouragement attacks;

5. Committee Manipulation Attacks: Mitigations: Given that committee selection is done in the blind, possibly even with trusted hardware, such attacks are virtually impossible;

6. Participants synching to a malicious chain after being offline for a long time: Mitigation to this attack is provided by the underlying consensus algorithm of the protocol.

\subsection{Proposal 2: Programmatic Value Recourse: Equitable Asset Participation}

Because it forms the socioeconomic backbone of a DAO, each commons requires a decentralized accounting system. In such an accounting system participants know that their contributions are recognized and valued fairly. This establishes and maintains trust, transparency, and strong reciprocity between DAO participants and encourages participation. This also applies to asset usage between DAOs expressed through their respective PC tokens because strong reciprocity creates efficient usage of existing assets and lets participants focus on adding value rather than recreating already existing value. It also encourages asset creators to share and combine assets within and across commons as the accounting system ensures sufficient protection. In surveying the limited literature, the concept of open value accounting (OVA) seems to have both an academic (Dunn et al. 2016; Haugen and Foster 2017) and a practical foundation (Sensorica n.d.). It is a framework that allows for resource and asset accounting for commons based peer production. At the ecosystem or DAO level, OVA describes the transformation and flow of resources within a DAO and with regard to asset and resource usage ascribed inside and outside a DAO. At the production level, it describes aggregation of resources and digital and analog contributions into a new asset or resource.

The OVA framework enables a DAO to track how contributions are evaluated and aggregated into assets such as products, services, infrastructure, social goods, etc. The OVA framework maintains a recording of who is doing what (contribution), how well (quality that can be translated into reputation and/or economic units), and how much (value) in a particular project. The framework outputs are a normalized distribution of benefits, including asset claims expressed as asset rights for the asset or resource output of a particular effort. In addition, economic gains generated by the asset or resource through a market or marketplace either within or outside the ecosystem are redistributed through the OVA framework based on the asset claims of the contributors.

Lastly, the OVA framework allows the re-appropriation of labor. It allows peer actors to turn their labor into asset claims and, hence, participate in the future economic gains such as revenue generated from the assets and resources they co-produce. Contribution tracking in terms of hours, resources, documentation, and designs etc., allows for the redistribution of benefits to project actors and incentivizes collaboration by reducing the risk of assets going "stale" due to a lack of sharing of know-how. Hence, the more people that have the know-how, the less the risk there is of a key person leaving a project and jeopardizing its continuation. This also assures people that their contributions are less likely to be wasted. The main components for the OVA are, therefore,

1. A Contributions Log to record actor contributions 
2. An Asset \& Resource Registry to create visibility of existing assets and resources to DAO actors

3. A Value Aggregation framework consisting of

a. A value equation and associated processes that describes how contributions are evaluated and associated with assets

b. Solidarity Mechanisms to ensure compassion and distribution of risk

4. A project marketplace where available projects are marketed and teams can be formed

To scale an OVA framework, we need to scale trust and, therefore, transparency and consensus. We propose to leverage existing components of a protocol layer and any employed digital identity protocol(s) of an ecosystem and secure consensus algorithms.

Each project in the DAO needs to define its own meaning of value. Then a process to assess the value of a contribution to an asset or deliverable needs to be defined. This can be unique to each project. Ideally, the DAO defines in its FBC guidelines how value is to be assessed (timeliness, resources expanded, deliverables met, quality requirements met, cost requirements met etc.). We will now show that even if every project has its own way to assess value, there will not be a strong dependence of on the way another project assesses value.

Once the definition of value has been settled, we recommend the following process to translate the assessed value into legal claim rights, such as percentage of future revenue to be received by an individual. The quantified value of the contribution is calculated based on the agreed to value equation. The validation vote of the contribution claim is effectively a vote for the calculated value. Value can be assessed in any combination of tokens ${ }^{27}$. If reputation tokens are chosen, the contribution has to be treated as a giving transaction ${ }^{28}$ and its evaluation should follow the process described in the section on reputation tokens in (Freund 2018). If other tokens such as (native) utility tokens of the underlying Blockchain platform or DAO tokens as normalizing measures are chosen, the assessed value is then translated into a claim right stake treated as an ownership stake, expressed through a token in the identity of the created asset. If there are multiple assets created in a project and combined into a single asset, or if multiple assets from within and outside a project are combined, the overall value contribution is assessed as follows (Note that we will use the word "owner" in the sense of a claims right holder of an asset):

1. An identity for the combined asset is created with the actors who have contributed to the asset as co-owners with ownership token amounts equivalent to the agreed upon value of their contributions;

2. The assets used to create the new asset are also made co-owners with ownership token amounts equivalent to the agreed upon value of the assets contribution. There are three options for assessing the value of the contribution. All three need to be functionally available in the three DAO commons:

a. The actors who have created the new asset assess the value of the parent assets without involvement of owners of the used assets. This is the most efficient solution. However, it is fraught with risk of one or more of the used asset owners, aka: claim rights holders filing a malicious action claim that could result in any number of adverse outcomes, such as loss of DAO stake, expulsion from the DAO, blacklisting, legal action etc.;

b. The actors who have created the new asset assess the value of the contribution as under (a) and then submit the value assessment within the PC to a vote of the owners of the

27 See the discussion in Section 3.1 on token types and how they might or might not be treated from a regulatory point of view.

28 A giving transaction is a cryptographic proof of a social good action that has not been done in exchange for any monetary value or other economically relevant advantage and is verifiable by a set of validators. See detailed definition of a giving transaction in (Freund 2018). 
used asset. The used asset owners can accept or reject the valuation. If it is rejected, the value assessment is referred to an arbitration function in the FBC containing the rejected value assessment and a proposed new value assessment. This arbitration function will be described in more detail below. Both parties are bound by the arbitration outcome;

c. The actors who have created the new asset assess the value of the used asset and submit it to the arbitration function of the FBC and the owners of the used asset. The process then follows the description under (b).

This approach will give visibility to the owners or claims right holders of an asset to all other assets that use it. This also ensures that a payment is made to the holder of asset claims in proportion to the PC token share, because the asset has generated value gain in terms of tokens, fiat or otherwise. Because the claim rights cascade through the nested asset identities two things can be achieved:

1. A claim holder can recursively call all assets where his or her asset is used and, therefore, construct a cryptographically verifiable claim structure that we are calling an identity graph. It should be noted here that in order to avoid payments to claims right holders in perpetuity as assets get fully commoditized, there should be a decay function over time in the number of claims rights held by an ecosystem participant in the form of tokens. This avoids the scenario of everyone still having to pay a small portion to the ancestors of the inventor of the wheel every time a wheel of any sort is sold;

2. The claims can be quantified and used to programmatically identify who needs to be compensated and how much in either Fiat or tokens through a Smart Contract payment system situated within the FBC.

\subsubsection{Attack Vectors \& Mitigations}

As with Proposal \#1 we need to discuss possible attack scenarios. Interestingly, and quite by design, the attack scenarios for Proposal \#2 are the same as for the application layer consensus algorithm discussed in Proposal \#1 because all of the DAO decision making processes are setting the rules and policies for equitable asset participation. Therefore, the same security guarantees discussed in Proposal \#1 apply here as well.

There is, however, one additional scenario that needs to be discussed. This is the scenario whena new policy does not have the desired effect for a significant portion of DAO participants and the vote to change these rules and policies fails, either maliciously or because a majority is experiencing a positive effect. In this scenario, the only recourse is to transfer assets and membership from one DAO to a new DAO. This outcome is very similar to choosing one chain over another in the discussion of collusion attacks in Proposal \#1.

\subsection{Proposal 3: Semi-Programmatic Monetary and Fiscal Token Policy}

In order to define and set monetary policy for protocol tokens such as Bitcoin and tokens defined on top of a protocol, such as ICO tokens on Ethereum, we need:

1. Policy Setting Mechanisms

2. Token Definitions and Policies

\subsubsection{Policy Setting Mechanisms}

We need to distinguish consensus models for their intended purpose. Is it required to keep a protocol such as Ethereum safe, or is it intended to reach agreement on the outcome of a predictions market? In the former case, the consensus model needs to secure an entire network. In the latter case, it only secures the value-at-risk for a particular prediction market.

To avoid delving into the computer science and economic complexities that are used to secure an entire network and where concepts such as rapidly reaching economic transaction finality are of prime 
importance ${ }^{29}$, we discuss a simplified consensus model taken from reference (Freund 2018) and used here for illustration. This model would be suitable for application layer token governance and allows it to reach consensus of an ecosystem outcome or state in a straightforward manner:

1. At the beginning of each ecosystem time period, a participant signals through a token stake, if they want to be an outcome or state validator;

2. Outcome/state specific rules are agreed upon by the affected counterparties. Ecosystem outcomes or states that require validation are assigned a random set of an uneven number of validators from the set of signaling validators. Signaling validators determine how many outcomes or states they want to validate. Validators need to stake each outcome/state they want to validate equally;

3. The assigned validators vote on the assigned ecosystem outcome/state during the voting period, which is determined ahead of time by the counterparties to the ecosystem outcome or state.

4. For an outcome or state to be validated, the validators in the consensus majority receive equal parts of the stake from the validators that were in the minority;

5. If the validators agree with the proposed outcome or state, the stake of the participant who wanted an outcome or state validated is returned. If not, then the stake is distributed in equal parts to the majority validators;

6. Any participant can raise an objection to the validator consensus in the ecosystem time period during, E, or immediately after, E + 1, the validator consensus was reached by requesting a 2nd round of validation by another set of randomly selected validators during the ecosystem time period, $\mathrm{E}+1$. In order to raise an objection, a participant needs to provide a stake for the objection in order to avoid spurious objections spamming the system. The size of the required stake is determined as a percentage of the value at stake; e.g., reputation tokens;

7. If the new set of validators agree with the objection, then the actor raising the objection receives the stake plus a percentage reward from the stake of the original giver and receiver as well as the stake of the initial set of validators. The new set of validators receives the remaining stake of the initial validators who were contradicted by the second set of validators. The exact percentages need to be specified either on the protocol level for utility services employing this approach or on a case-by-case basis at the application layer. In both instances, the consensus process parameters are determined by consensus of the participating actors;

8. If the new set of validators disagree with the objection, then the objecting actor loses its stake. The stake is distributed in equal parts amongst the validators that confirmed the conclusion of the original validator set.

\subsubsection{Token Definitions and Policies}

We describe general token characteristics required for monetary and fiscal policy making below. Tokens might have other qualities such as representing units of $\mathrm{CO}_{2}$ saved or produced or a laundromat service. Note that we will call out protocol tokens and application level tokens separately where required:

1. Through consensus controlled changes by ecosystem participants, it should be possible to add additional token types to an ecosystem, including at the protocol level. The type of tokens should be unconstrained. This allows the necessary flexibility to create new token types required as the ecosystem evolves, such as reputation;

2. The initial types of tokens at the protocol level cannot be retired, in other words being taken fully out of circulation. This ensures security against economic extortion or discouragement attacks at

29 Economic Finality: A state H1 is economically finalized if enough validators sign a message attesting to H1, with the property that if both $\mathrm{H} 1$ and a conflicting $\mathrm{H} 2$ are finalized, then there is evidence that can be used to prove that at least $\mathrm{x} \%$ of validators were malicious and therefore destroy their entire deposits where $\mathrm{x}=1-p$ with $p>50 \%$ being the consensus majority parameter see Casper FFG (Buterin and Griffith 2017) as an example. 
a platform level. Any additional protocol token types created in addition to the initial base tokens can be retired and taken out of circulation, as long as a majority consensus of token holders agrees on a retirement proposal. The exact consensus criteria for token retirement or any token utility changes have to be implemented at the token protocol level, as part of the overall ecosystem protocol, and should not be changeable after initiation to avoid extortion and discouragement attacks on a token economy. Therefore, the consensus rules have to be carefully crafted at the beginning to allow for sufficient fiscal and monetary token flexibility. For example, voting rights based strictly on the size of stake will lead to power concentration in the token economy, which is to be avoided at all cost as this leads to self-optimization rather than ecosystem-optimization;

3. A token should allow a participant to participate in any ecosystem actions; e.g., buying and selling of goods and services;

4. A protocol token does not represent an ownership right. It represents a unit of ecosystem economic value, at minimum for utility services. Ownership of assets should be managed through tokens defined at the application layer. Assets themselves are to be defined at the application and not the protocol layer;

5. One or more tokens can be earned through actions in an ecosystem; e.g., services for tokens that are governed by a set of rules agreed upon by the counterparties of the action;

6. Except for reputation tokens, tokens should be freely transferable amongst ecosystem participants;

7. Except for reputation tokens, tokens are freely exchangeable for any other token and any other non-ecosystem token (cross ecosystem token transfer e.g., Token to Ether or USD);

8. There should be a fixed number of tokens at ecosystem creation (ecosystem specific and agreed upon by ecosystem instantiating actors) with the number allowed to increase or decrease over time as mentioned before;

9. New tokens at the protocol level are created only through utility services such as running a Blockchain node. The number of new tokens per service; e.g., the size of a Block reward—and what constitutes a utility service is agreed upon by the participants defining a utility service for an ecosystem through consensus;

10. New tokens are distributed to one or more participants providing an ecosystem utility service. The manner of distribution is specific to an ecosystem and is agreed upon by the participants in utility services through consensus; e.g., based on the size of their stake in a PoS consensus model powering a Blockchain;

11. The growth of a token quantity over one or more fixed time periods should be limited to $Y \%$. The growth rate $Y$, the number of time periods, and the definition of length of a time period is governed by consensus of the ecosystem participants;

12. Tokens can be destroyed through the actions of participants either voluntarily or involuntarily. The rules governing the destruction of tokens require:

a. Consensus of ecosystem participants if the rules can affect the token balance of any actor in an ecosystem, such as ecosystem utility services.

b. One or more ecosystem participants if the destruction rules affect only the token balances of ecosystem participants making the token destruction rules. A token contract governing a token will have to contain a method that allows a participant holding tokens to destroy them. In order to avoid malicious behavior in case a participant is compromised, there should be an escrow function as part of the token contract that either prevents or makes malicious behavior very difficult.

\subsubsection{Attack Vectors \& Mitigations}

Interestingly enough since consensus algorithms are also the pillars of Proposal \#3, the same arguments applied to Proposal \#2 apply also to Proposal \#3. 


\subsection{Proposal 4: Decentralized Exchanges}

To avoid the destabilizing scenarios of centralized exchanges, we suggest a decentralized exchange facility as a programmatic ecosystem utility service ${ }^{30}$, ideally at the platform layer, which simplifies operations; all platform participants pay for its maintenance through fees and block rewards ${ }^{31}$. The decentralized exchange facility should be designed:

- $\quad$ where there are no information and trading capability asymmetries due to full exchange market transparency and standard trading capabilities provided to everyone.

- so the exchange governance itself is being administered through a DAO/Common with built-in programmatic consensus mechanisms.

- where liquidity cannot be artificially concentrated since non-ecosystem assets used in the exchange facility are required to be escrowed, increasing the opportunity cost of external assets if only used for extractive purposes (short term horizon).

- where token values of a decentralized economy are, at least partially, tied to underlying digital assets rather than just based on the trust external actors place in the DAO governing the decentralized economy/commons.

This last point requires an additional word of explanation. If we look at the history of currencies that were tied to gold as the underlying reserve asset we gain important insights: History shows that in times of significant value decline, such as during the Great Depression, or significant value growth, such as after WWII, coupling a currency to gold was a disadvantage. In the Great Depression devaluation of currency after partial decoupling from gold was successfully used to relieve the debt load. And after WWII (partial) decoupling from gold was successfully used to account for significant value increases from rapid growth of the labor force and process automation both taking place at a faster pace than gold could be physically mined. In other words, being tied to an "inflexible" asset class as a reserve lacked the required monetary flexibility. Therefore, the tie to gold was dissolved over time, which Bretton-Woods finally abandoned in the 70s, and replaced by the financial trustworthiness of countries themselves as the "intangible" reserve asset. This trust represented a significantly higher value reserve than gold as it was much more flexible; at least in the opinion of investors. At the same time, this trust is unfortunately not accessible to a true accounting system as gold was, thereby, allowing "fuzziness" in terms of asset accounting and evaluation. This lack of quantifiable accounting and valuation led straight to the Global Financial Crisis because values of highly leveraged assets were based on wrong trust assumptions, such as default rates.

Coming back to gold as a currency reserve asset, in times other than that of rapid change as described above, the tie to gold provided significant currency stability. Since decentralized economies, especially emerging ones, do not have financial trust in the traditional sense, they should not be subject to "fuzzy", non-objective value measures based on the opinion of a few financial experts such as rating agencies, but rather employ transparent asset accounting systems that are censorship resistant and tamper proof as we discussed above with the OVA framework. Therefore, we should be looking for ways to combine the best of both worlds, the stability that tying a currency to a highly valued asset class provides, while at the same time having flexibility in the reserve asset class itself to either increase or decrease its volume and value-imagine you could easily mine or destroy gold.

Digital assets as a reserve asset in a decentralized economy have the advantage that they can be increased or decreased in volume and/or distributed below market value, if owned by an asset common, based on programmatically enforced participant consensus. An example of such an asset is open source software. The issue with open source software is that there is currently no way to accurately and quantitatively account for value contributions by individuals to an open source project.

30 See (Freund 2018) for a detailed description and discussion of such a decentralized exchange facility and its processes.

31 See (Freund 2018) for a detailed description and discussion of such a decentralized exchange facility and its processes. 
Therefore, subsequent monetization of the open source asset by third parties without reciprocity to the contributors to an asset common, such as an open source project, is currently the norm and not the exception.

Leveraging Blockchain technology, we are now able to programmatically not only provide an open, censorship resistant, and tamper proof value accounting system, but are also able to freely define and value assets based on perceived value by participants. In addition, we can define programmatically enforced rules around asset usage and exchanges such that, for example, the monetization of an open source project from a decentralized economy is appropriately shared with the producing common. This ensures reciprocity between asset user and creator.

\subsubsection{Attack Vectors \& Mitigations}

The same arguments used in Proposal \#1 and applied to Proposal \#2 and to Proposal \#3, apply here as well for the exchange policies to be set. There are, however, a few nuances that allow for additional attack vectors, which we will discuss below in more detail:

1. Front Running/Transaction Ordering Attacks: This is a quite simple, yet effective attack. A Blockchain node can observe all bid/ask spreads in the exchange as well as all incoming exchange transactions. With this information, an attacker controlling a Blockchain node can exploit the information asymmetry between what the node can see and the other market participants and create arbitrage by placing its own exchange transaction before all others and, therefore, capitalize on small price imbalances. Mitigation: As discussed in Proposal \#1 and detailed in (Bakshi and Freund 2018), these type of attacks can be mitigated using consensus driven overlay networks using trusted hardware;

2. Transaction Censoring Attacks: Besides inserting their own transaction ahead of any other exchange transaction, a malicious node can simply ignore transactions and never execute them. We have discussed this extensively before in Proposal \#1. Mitigation: The mitigation strategy is the same as for Front Running/Transaction Ordering Attacks;

3. Value Extraction Attacks: Such attacks can take many forms, such as hording tokens, short selling tokens in secondary markets not controlled by the decentralized exchange, or otherwise trying to manipulate prices through collusion and/or extortion attacks unrelated to consensus. Mitigation: To avoid such value extraction attacks the DAO regulating the decentralized exchange facility can increase capital requirements to be escrowed, increasing the opportunity costs of attackers significantly. The DAOs that have issued tokens that are traded can use Proposal \#3 and increase token supply when for example hording is detected, which will bring down token prices. Short selling of tokens is also not possible since one has to lock up capital to do so. This capital could be totally lost if a malicious action proposal, see (Freund 2018) for more details, is submitted and accepted to the DAO overseeing the decentralized exchange functionality. Again, the full transparency of the ledger prevents malicious actors to leverage information asymmetry for gains.

\section{Conclusions}

We recognize that we are observing a societal shift from competitive marketplaces, populated largely by centralized organizations, to decentralized socioeconomic markets. We believe that existing research indicates that this is happening because life conditions cause values and priorities to shift. These new markets are rising out of existing competitive markets where socioeconomics have been taken into stronger consideration by borrowing characteristics from historical cooperative business models and the newer social enterprise, effectively combining commercial and social elements. These models are being pioneered by internet and software mediated networks like Sensorica, Fairmondo, Cocoon Projects, Enspiral, MakeSense, and OuiShare (Manzanedo and Trepat 2017). 
Blockchain is the foundational technology in the next wave of decentralized socioeconomic models like Backfeed, Colony.io, district0x, and Peerism.

As decentralized socioeconomic models begin to develop, they must survive within the existing competitive markets environment. We discussed how competitive markets are incented to extract value from any source because of their socially accepted and singular definition of value, while decentralized socioeconomic markets are incented to generate value towards their common purpose as the result of a freedom to self-define value. This ability to self-define value is both their greatest differentiator and weakness. At startup, their new value regime is not widely trusted, and having a commons structure makes them vulnerable to extractive forces accessing free resources without reciprocating value. In addition, the currencies of decentralized economies are vulnerable to arbitrage attacks originating from lack of liquidity, trust asymmetry, unintended use of the currency, and slow reactions to attacks. These vulnerabilities motivated our discussion of case studies showing designed market interfaces that attempt to protect value sovereignty. The lessons learned from those, and other designs helped inform our recommendation for a three zone model to create resilient decentralized models, and the four proposals for protecting decentralized economies using decentralized ledger technologies like Blockchain.

Proposal \#1 is for collusion resistant and tamper proof consensus governance that provides timely decision making within decentralized groups. It is designed to avoid actors gaining enough power to negatively impact the decentralized economy's value regime. This consensus mechanism allows the decentralized economy to adjust rewards and fee schedules that control a token's fiscal and monetary policy. This avoids issues seen in the Ecuador case study where decisions were not made in a timely fashion, hence most of the proposals were not enforced, and the vulnerability of Enspiral where all governance decisions are made on centralized software.

Proposal \#2 is for programmatic value recourse requiring equitable asset participation. This is closely modeled after the open value accounting system of Sensorica, but solves the problem of external agreements of reciprocity being shirked. This proposal lays out an accounting system that tracks all contributions to an asset, digital and analog. When an asset is monetized, that value is redistributed to all contributors based on their agreement of the value each contributor should earn.

Proposal \#3 is semi-programmatic monetary and fiscal policy. It is a consensus process for app layer token governance. It is designed for rapid but secure decision making for adjusting monetary and fiscal policy. This solves what we consider to be a problem with FairCoin's permanently static coin supply. This proposal also gives members the ability to add new tokens, but restricts their ability to destroy any initial token completely or any other tokens without majority consensus from those it would affect. This is also an improvement from FairCoin, where the monetary and fiscal policies are governed by an elected assembly of people, centralizing power into what could be a relatively small group compared to all members.

Proposal \#4 is a decentralized exchange, which we consider to be an improvement of FairCoin's exchange so that information asymmetry is not exploited in exchange, negatively impacting the token's value. Proposal \#4 stipulates that governance of the exchange is consensus driven. It establishes liquidity by tying the tokens to digital or analog resources that are quantified assets with stable value over time (like gold) with flexible reserve amounts (unlike gold).

These proposals are improvements upon what has been observed in different real-world market interface designs. They are, therefore, theoretical and untested, but based on empirical and theoretical research. They will be most effective in protecting decentralized socioeconomic markets when used together, but can provide incremental improvements when applied separately.

Author Contributions: A.F. conceived of the three zone model and market interface working proposal to protect decentralized economies. D.S. framed and analyzed the competitive and decentralized socioeconomic market structures, identified and analyzed value extraction attacks, and identified and analyzed the case studies. A.F. and D.S. co-authored the paper. 
Acknowledgments: The authors would like to express their deepest gratitude to all the open source reviewers of this paper, in particular our colleagues David Kish, Justin Kersey, and Ashley Bremer. Both authors are employees of Tata Consultancy Services LLC and want to express appreciation to our employer for letting us carry out this work. This paper reflects our own opinions and is not representative of those of our employer, Tata Consultancy Services LLC.

Conflicts of Interest: The authors declare no conflict of interest. The funding sponsor had no role in the design of the study; in the collection, analyses, or interpretation of data; in the writing of the manuscript, and in the decision to publish the results.

\section{References}

Akerlof, George A. 1995. The Market for 'Lemons': Quality Uncertainty and the Market Mechanism. In Essential Readings in Economics. Edited by Saul Estrin and Alan Marin. London: Macmillan Education UK, pp. 175-88.

Arnold, Malcolm F. 2008. Non-financial performance metrics for corporate responsibility reporting revisited. A Doughty Centre for Corporate Responsibility. Working paper, 40. Available online: https:/ / dspace.lib. cranfield.ac.uk/handle/1826/3222 (accessed on 4 May 2018).

Our Product-B3i. n.d. Available online: https://b31tech/our-product.html (accessed on 7 May 2018).

Backfeed I Spreading Consensus. n.d. Available online: http://backfeed.cc/ (accessed on 4 May 2018).

Bakshi, Sanjay, and Andreas Freund. 2018. Increasing Blockchain Security, Scalability, Privacy and Decentralization through Trusted Compute Resources. Preprint in preparation.

Bano, Shehar, Alberto Sonnino, Mustafa Al-Bassam, Sarah Azouvi, Patrick McCorry, Sarah Meiklejohn, and George Danezis. 2017. SoK: Consensus in the Age of Blockchains. Available online: https://arxiv.org/pdf/ 1711.03936 (accessed on 7 May 2018).

Bauwens, Michel, and Vasilis Niaros. 2016. Value in the Commons Economy: Developments in Open and Contributory Value Accounting. Available online: http:/ / confrontations.org/wp-content/uploads/2016/ 12/1.Value-in-the-Commons-Economy_M.-Bauwens.pdf (accessed on 15 March 2018).

Bauwens, Michel, and John Restakis. 2015. Commons Transition: Policy Proposals for an Open Knowledge Commons Society. Available online: https://commonstransition.org/wp-content/uploads/2014/11/ Commons-Transition_-Policy-Proposals-for-a-P2P-Foundation.pdf (accessed on 1 March 2018).

Beck, Don Edward, Teddy Hebo Larsen, Sergey Solonin, Rica Viljoen, Rica Viljoen, and Thomas Johns. 2018. Spiral Dynamics in Action: Humanity's Master Code. Hoboken: Wiley. Available online: https://books.telegraph.co. uk/Product/Don-Edward-Beck/Spiral-Dynamics-in-Action--Humanitys-Master-Code/20666740 (accessed on 9 May 2018).

Bissonnette, Zac. 2015. The Great Beanie Baby Bubble by Zac Bissonnette. Portfolio/Penguin. Available online: https:www.penguinrandomhouse.combooks313121the-great-beanie-baby-bubble-by-zacbissonnette9781591848004 (accessed on 9 May 2018).

Blenko, Marcia, Michael C. Mankins, and Paul Rogers. 2010. Decide E Deliver: 5 Steps to Breakthrough Performance in Your Organization. Boston: Harvard Business Review Press.

Boldrin, Michele, and David K. Levine. 2005. Intellectual Property and the Efficient Allocation of Social Surplus from Creation. Review of the Economic Research on Copyright Issues 2: 45-66.

Bollier, David. 2017. Re-Imagining Value: Insights from the Care Economy, Commons, Cyberspace and Nature. Commons Strategies Group in cooperation with the Heinrich Böll Foundation and David Graeber. Available online: https://www.boell.de/en/2017/03/07/re-imagining-value-insights-careeconomy-commons-cyberspace-and-nature (accessed on 22 March 2018).

Boschee, Jerr. 2010. Merging the Profit Motive and Moral Imperatives: The Rise of Social Enterprise in the United States. Geneva: The Institute for Social Entrepreneurs.

Brown, Dana. 2017. Ecuador's 'Citizen Revolution.' Latin America's "Pink Tide" and the Challenge of Systemic Change (Blog). Available online: https://thenextsystem.org/learn/stories/ecuadors-citizen-revolution (accessed on 7 August 2017).

Buterin, Vitalik, and Virgil Griffith. 2017. Casper the Friendly Finality Gadget. ArXiv.

Coindesk. 2018. Available online: https://www.coindesk.com/vietnam-investigates-ico-fraud-660-millionlosses-reported/ (accessed on 11 April 2018).

Colony: A Platform for Open Organizations. n.d. Available online: https:/ / colony.io (accessed on 7 May 2018). 
Commons Transition Primer. 2018. Case Studies. Available online: http://primer.commonstransition.org/4more/5-elements/case-studies (accessed on 15 March 2018).

Commons-Based Peer Production Directory. 2018. Welcome to the P2Pvalue Directory of Commons Based Peer Production. Available online: http:/ / directory.p2pvalue.eu/ (accessed on 15 March 2018).

Cong, Lin William, and Zhiguo He. 2018. Blockchain Disruption and Smart Contracts. Available online: https: / / www.ssrn.com/abstract=2985764 (accessed on 9 May 2018).

Cong, Lin William, Zhiguo He, and Jiasun Li. 2018. Decentralized Mining in Centralized Pools. Available online: https: / / www.ssrn.com/abstract=3143724 (accessed on 9 May 2018).

Dave Grace \& Associates. 2014. Measuring the Size and Scope of the Cooperative Economy: Results of the 2014 Global Census on Co-Operatives. For the United Nation's Secretariat Department of Economic and Social Affairs Division for Social Policy and Development. Available online: http:/ /www.un.org/esa/socdev/ documents/2014/coopsegm/grace.pdf (accessed on 22 March 2018).

Dees, Gregory, and Beth Battle Anderson. 2007. Enterprising Social Innovation: Focusing Research on the Most Intriguing Form of Social Entrepreneurship. Social Enterprise Reporter 302-3: 1-14.

Digital Library of the Commons. n.d. Available online: http://dlc.dlib.indiana.edu/dlc/contentguidelines (accessed on 22 March 2018).

District0x. n.d. Available online: https:/ / district0x.io/ (accessed on 7 May 2018).

Dunbar, Robin I. M. 1992. Neocortex Size as a Constraint on Group Size in Primates. Journal of Human Evolution 22: 469-93. [CrossRef]

Dunn, Cheryl, Gregory Gerard, and Severin Grabski. 2016. Resources-Events-Agents Design Theory: A Revolutionary Approach to Enterprise System Design. Communications of the Association for Information Systems 38. [CrossRef]

DuPont, Quinn. 2017. Experiments in Algorithmic Governance: A History and Ethnography of "The DAO," a Failed Decentralized Autonomous Organization. Chapter 8, Bitcoin and Beyond. Abingdon: Routledge. Available online: https://www.researchgate.net/publication/319529311_Experiments_in_Algorithmic_Governance_ A_history_and_ethnography_of_The_DAO_a_failed_Decentralized_Autonomous_Organization (accessed on 9 May 2018).

Easley, David, Maureen O'Hara, and Soumya Basu. 2017. From Mining to Markets: The Evolution of Bitcoin Transaction Fees. Available online: https: / / www.ssrn.com/abstract=3055380 (accessed on 9 May 2018).

Faircoin-P2P Foundation. n.d. Available online: http://wiki.p2pfoundation.net/Faircoin\#Details (accessed on 22 March 2018).

FairCoop. 2017. What Can We Do with Our FairCoin? Available online: https:/ / fair-coin.org/en/what-can-wedo-our-faircoin (accessed on 22 March 2018).

FairCoop principles. n.d. Available online: https:/ /2017.fair.coop/principles/ (accessed on 9 May 2018).

FairFunds. n.d. Available online: https:/ /2017.fair.coop/fairfunds/ (accessed on 9 May 2018).

Filippi, Primavera De, Benjamin Loveluck, Vasilis Niaros, Alekos Pantazis, and Mayo Fuster Morell. n.d. Best Practices for CBPP Communities \& Policy Recommendations. Available online: https:/ / p2pvalue.eu/wpcontent/uploads/2013/07/Deliverable_2.3-updated.pdf (accessed on 22 March 2018).

Financial Agreement Enspiral Handbook. n.d. Available online: https:/ /handbook.enspiral.com/agreements / financial.html (accessed on 9 May 2018).

Freund, Andreas. 2018. Model for Consensus Controlled, Socioeconomic Incentivized and Platform Driven Decentralized Economies and Businesses. Available online: https://docs.google.com/document/d/ 1vlOoXhHIS2hSNysH7eP44X3yP990F0zHMV_rSztQGZs/edit (accessed on 9 May 2018).

Giotitsas, Chris, and Jose Ramos. 2017. A New Model of Production for a New Economy. The New Economics Foundation. Available online: https:/ / p2pfoundation.net/wp-content/uploads/2017/09/A-New-Modelof-Production-for-a-New-Economy-FINAL.pdf (accessed on 22 March 2018).

Goldgar, Anne. 2007. Tulipmania: Money, Honor, and Knowledge in the Dutch Golden Age. Available online: http: / / www.press.uchicago.edu/ucp/books/book/chicago/T/bo5414939.html (accessed on 22 March 2018).

Graves, Clare W. 2005. The Never Ending Quest: Dr. Clare W. Graves Explores Human Nature: A Treatise on an Emergent Cyclical Conception of Adult Behavioral Systems and Their Development. ECLET Publishing. Available online: https://www.alibris.com/The-Never-Ending-Quest-Dr-Clare-W-GravesExplores-Human-Nature-a-Treatise-on-an-Emergent-Cyclical-Conception-of-Adult-Behavioral-Systemsand-Their-Development/book/-97247421 (accessed on 22 March 2018). 
Harriman, Alyssa. 2015. The Making of a Movement: The Rise of the B Corp on the Global Stage. Ph.D. thesis, Copenhagen Business School, Frederiksberg, Denmark, p. 103.

Haugen, Bob, and Lynn Foster. 2017. ValueFlows. Available online: https:/ /www.valueflo.ws/ (accessed on 22 March 2018).

Huberman, Gur, Jacob D. Leshno, and Ciamac C. Moallemi. 2017. Monopoly without a Monopolist: An Economic Analysis of the Bitcoin Payment System. Available online: https://www.ssrn.com/abstract=3025604 (accessed on 22 March 2018).

ICOdata.io. 2018. Available online: icodata.io/stats/ (accessed on 9 May 2018).

Kish, David. n.d. Ecosystem of You Presentation. The Next Evolution (Blog). Available online: http:/ / www. thenextevolution.com/presentations-2/ecosystem-of-you-presentation/ (accessed on 22 March 2018).

König, Thomas. 2016. FairCoin V2 White Paper. Available online: https://chain.fair-coin.org/download/ FairCoin2-white-paper-V1.1.pdf (accessed on 9 May 2018).

Kurzweil, Ray. 2005. Singularity Is Near. Penguin Random House. Available online: https:www. penguinrandomhouse.combooks291221pp-singularity-is-near-by-ray-kurzweil (accessed on 22 March 2018).

Lewis, Michael. 2014. The New New Thing: A Silicon Valley Story. New York: W. W. Norton \& Company.

Li, Jiasun, and William Mann. 2018. Initial Coin Offering and Platform Building. Available online: https: / / papers.ssrn.com/sol3/papers.cfm?abstract_id=3088726 (accessed on 9 May 2018).

Manzanedo, Ana, and Alícia Trepat. 2017. Designing Positive Platforms: A Guide for a Governance-Based Approach. Palo Alto: Institute for the Future Research on Positive Platforms, p. 16.

Mueller, Lukas. 2018. Conceptual Framework for Legal and Risk Assessment of Crypto Tokens. Available online: https:/ / www.mme.ch/fileadmin/files/documents/180501_BCP_Framework_for_Assessment_of_ Crypto_Tokens_-_Block_2.pdf (accessed on 9 May 2018).

National Association of Housing Cooperatives. n.d. Significant Dates in Cooperative History. Available online: https: / / coophousing.org/resources/general-cooperative-information/significant-dates-in-cooperativehistory/ (accessed on 22 March 2018).

Ortmann, Gerald F., and Rober P. King. 2007. Agricultural Cooperatives I: History, Theory and Problems. Agrekon 46: 18-46. [CrossRef]

Pass, Rafael, and Elaine Shi. 2016a. Snow White: Provably Secure Proofs of Stake. Available online: https: / / ia.cr/2016/919 (accessed on 9 May 2018).

Pass, Rafael, and Elaine Shi. 2016b. The Sleepy Model of Consensus. Available online: https://ia.cr/2016/918 (accessed on 9 May 2018).

Peer Production License-P2P Foundation. n.d. Available online: http://wiki.p2pfoundation.net/Peer_Production_ License\#Simple_Definition_and_the_PPL_as_a_Transvestment_Stategy (accessed on 22 March 2018).

Peerism: The Skill Token Economy for Post-Capitalism. n.d. Available online: http:/ / peerism.org/ (accessed on 7 May 2018).

Poon, Daryl. 2011. The Emergence and Development of Social Enterprise Sectors. Available online: https: / repository.upenn. edu/cgi/viewcontent.cgi?referer=https: / / www.google.com/\&httpsredir=1\&article=1010\&context=sire (accessed on 9 May 2018).

R3.Com Technology. n.d. Available online: https:/ / www.r3.com/technology / (accessed on 7 May 2018).

Saleh, Fahad. 2017. Blockchain without Waste: Proof-of-Stake. Available online: https:/ / pdfs.semanticscholar. org/cd35/131e29c6122dbacdb95d569be5b391da083a.pdf (accessed on 9 may 2018).

Sensorica. n.d. Sensorica-Commons Transition Wiki. Available online: http:/ /wiki.commonstransition.org/ wiki/Sensorica\#Value_Accounting_in_Sensorica (accessed on 22 March 2018).

Smith, H. Jeff. 2003. The Shareholders vs. Stakeholders Debate. Magazine. MIT Sloan Management Review (Blog). Available online: https://sloanreview.mit.edu/article/the-shareholders-vs-stakeholders-debate/ (accessed on 22 March 2018).

Startup Tracker. 2018. Outlier Ventures (Blog). Available online: https://outlierventures.io/startup-tracker/ (accessed on 16 February 2018).

Sundell, Ville. 2017. "Etherpriseses LLC, set up your own company on Ethereum". Available online: https: / / www.linkedin.com/pulse/etherprises-llc-set-up-your-own-company-ethereum-ville-sundell (accessed on 9 May 2018).

Torrance, Andrew W., and Bill Tomlinson. 2009. Patents and the Regress of Useful Arts. The Columbia Science and Technology Law Review 10: 130-68. 
Venture Agreement Enspiral Handbook. n.d. Available online: https:/ /handbook.enspiral.com/agreements / venture.html (accessed on 9 May 2018).

Yermack, David. 2016. Corporate Governance and Blockchain. Available online: www.nber.org/papers/w21802 (accessed on 9 May 2018). 